\title{
Learning Mixtures of Linear Regressions in Subexponential Time via Fourier Moments
}

\author{
Sitan Chen* \\ EECS, MIT \\ USA \\ sitanc@mit.edu
}

\author{
Jerry Li \\ Microsoft Research \\ USA \\ jerrl@microsoft.com
}

\author{
Zhao Song \\ Princeton \& IAS \\ USA \\ zhaos@ias.edu
}

\begin{abstract}
We consider the problem of learning a mixture of linear regressions (MLRs). An MLR is specified by $k$ nonnegative mixing weights $p_{1}, \ldots, p_{k}$ summing to 1 , and $k$ unknown regressors $w_{1}, \ldots, w_{k} \in \mathbb{R}^{d}$. A sample from the MLR is drawn by sampling $i$ with probability $p_{i}$, then outputting $(x, y)$ where $y=\left\langle x, w_{i}\right\rangle+\eta$, where $\eta \sim \mathcal{N}\left(0, \varsigma^{2}\right)$ for noise rate $\varsigma$. Mixtures of linear regressions are a popular generative model and have been studied extensively in machine learning and theoretical computer science. However, all previous algorithms for learning the parameters of an MLR require running time and sample complexity scaling exponentially with $k$.

In this paper, we give the first algorithm for learning an MLR that runs in time which is sub-exponential in $k$. Specifically, we give an algorithm which runs in time $\widetilde{O}(d) \cdot \exp (\widetilde{O}(\sqrt{k}))$ and outputs the parameters of the MLR to high accuracy, even in the presence of nontrivial regression noise. We demonstrate a new method that we call Fourier moment descent which uses univariate density estimation and low-degree moments of the Fourier transform of suitable univariate projections of the MLR to iteratively refine our estimate of the parameters. To the best of our knowledge, these techniques have never been used in the context of high dimensional distribution learning, and may be of independent interest. We also show that our techniques can be used to give a sub-exponential time algorithm for a natural hard instance of the subspace clustering problem, which we call learning mixtures of hyperplanes.
\end{abstract}

\section{CCS CONCEPTS}

- Theory of computation $\rightarrow$ Unsupervised learning and clustering; • Mathematics of computing $\rightarrow$ Probability and statistics.

\section{KEYWORDS}

Mixture models, linear regression, unsupervised learning, Fourier analysis, method of moments

\footnotetext{
*This work was supported in part by a Paul and Daisy Soros Fellowship, NSF CAREER Award CCF-1453261, and NSF Large CCF-1565235. This work was done in part while S.C. was an intern at Microsoft Research AI.

Permission to make digital or hard copies of all or part of this work for personal or classroom use is granted without fee provided that copies are not made or distributed for profit or commercial advantage and that copies bear this notice and the full citation on the first page. Copyrights for components of this work owned by others than ACM must be honored. Abstracting with credit is permitted. To copy otherwise, or republish, to post on servers or to redistribute to lists, requires prior specific permission and/or a fee. Request permissions from permissions@acm.org.

STOC '20, June 22-26, 2020, Chicago, IL, USA

(C) 2020 Association for Computing Machinery.

ACM ISBN 978-1-4503-6979-4/20/06 . \$ \$15.00

https://doi.org/10.1145/3357713.3384333
}

ACM Reference Format:

Sitan Chen, Jerry Li, and Zhao Song. 2020. Learning Mixtures of Linear Regressions in Subexponential Time via Fourier Moments. In Proceedings of the 52nd Annual ACM SIGACT Symposium on Theory of Computing (STOC '20), June 22-26, 2020, Chicago, IL, USA. ACM, New York, NY, USA, 14 pages. https://doi.org/10.1145/3357713.3384333

\section{INTRODUCTION}

Mixtures of linear regressions (or MLRs for short) are a popular generative model, and have been studied extensively in machine learning and theoretical computer science. A standard formulation of the problem is as follows: we have $k$ unknown mixing weights $p_{1}, \ldots, p_{k}$ which are non-negative and sum to $1, k$ unknown regressors $w_{1}, \ldots, w_{k} \in \mathbb{R}^{d}$, and a noise rate $\varsigma \geq 0$. A sample from the MLR is drawn as follows: we first select $i \in[k]$ with probability $p_{i}$, then we receive $(x, y)$ where $x \in \mathbb{R}^{d}$ is distributed as $\mathcal{N}(0, \mathrm{I})$ and $y=\left\langle w_{i}, x\right\rangle+\eta$, where $\eta \sim \mathcal{N}\left(0, \varsigma^{2}\right)$. This model has applications to problems ranging from trajectory clustering [19] to phase retrieval $[4,5,36]$ and is also widely studied as a natural non-linear generative model for supervised data [3, 6, 10, 17, 27-30, 40, 46-48].

The basic learning question for MLRs is as follows: given i.i.d. samples $\left(x_{1}, y_{1}\right), \ldots,\left(x_{n}, y_{n}\right) \in \mathbb{R}^{d} \times \mathbb{R}$ from an unknown MLR, can we learn the parameters of the underlying MLR? To ensure that the parameters are identifiable, it is also typically assumed that the regressors are separated in some way, e.g. there is some $\Delta>0$ so that $\left\|w_{i}-w_{j}\right\|_{2} \geq \Delta$ for all $i \neq j$.

Despite the apparent simplicity of the problem, efficiently learning MLRs given samples has proven to be a surprisingly challenging task. Even in the special case where $\varsigma=0$, that is, we assume that there is no noise on the samples, the fastest algorithms for this problem run in time depending on $k^{\Omega(k)}$ [30,48]. It turns out that there are good reasons for this barrier.

Previous algorithms for this problem with end-to-end provable guarantees-and indeed, the vast majority of statistical learning algorithms in general-build in some form or another on the method of moments paradigm. At a high level, these methods require that there exists some statistic which depends only on low degree moments of the unknown distribution, so that a sufficiently good estimate of this statistic will uniquely identify the parameters of the distribution. This includes widely-used techniques based on tensor decomposition [6, 40,47,48], and SDP hierarchies such as the Sum-of-Squares meta-algorithm [26, 39]. If degree $t$ moments are necessary to devise such a statistic, then these methods require $\exp (\Omega(t))$ sample and computational complexity.

Unfortunately, for MLRs, it is not hard to demonstrate pairs of mixtures some of whose parameters are far apart from each other, where all moments of degree at most $2 k-1$ of the two mixtures 
agree exactly (see the full version for more details). As a result, any moment-based estimator would need to use moments of degree at least $\Omega(k)$, and hence require a runtime of $\exp (\Omega(k))$. This imposes a natural bottleneck: any algorithm that hopes to achieve sub-exponential time must somehow incorporate additional information about the geometry of the underlying learning problem.

A related problem, which shares a similar bottleneck, is the problem of learning mixtures of Gaussians under the assumption of angular separation. A concrete instantiation of this problem is a model we call learning mixtures of hyperplanes. A mixture of hyperplanes is parameterized by mixing weights $p_{1}, \ldots, p_{k}$, a separation parameter $\Delta>0$, and $k$ unit vectors $v_{1}, \ldots, v_{k}$ satisfying $\left\|v_{i} \pm v_{j}\right\|_{2} \geq \Delta$ for all $i \neq j$ (note that the reason for the \pm is that the directions of a mixture of hyperplanes are only identifiable up to sign). To draw a sample, we first draw $i \in[k]$ with probability $p_{i}$, and then draw a sample from $\mathcal{N}\left(0, \mathbf{I}-v_{i} v_{i}^{\top}\right)$.

As before, the corresponding learning question is: given samples from an unknown mixture of hyperplanes, can one recover the underlying parameters? This problem can be thought of as a particularly hard case of the well-studied problem of subspace recovery, where current techniques require time exponential in $k$.

In this paper, we give algorithms which are able to achieve strong recovery guarantees for the problems of learning MLRs and learning mixtures of hyperplanes, and which run in time which is subexponential in $k$. To the best of our knowledge, this is the first algorithm for the basic problem of learning MLRs which achieves sub-exponential runtime without placing strong additional assumptions on the model. At a high level, our key insight is that while low degree moments of the MLR are unable to robustly identify the instance, low degree moments of suitable projections of the Fourier transform of the MLR can be utilized to extract non-trivial information about the regressors. We then give efficient algorithms for computing such "Fourier moments" by leveraging algorithms for univariate density estimation $[1,7]$. This allows us to dramatically improve the runtime and sample complexity of the moment descent algorithm of [30], and allows us to obtain our desired subexponential runtime. We believe that this sort of algorithmic application of the continuous Fourier transform and of univariate density estimation to a high dimensional learning problem is novel, and may be of independent interest.

\subsection{Our Contributions}

Here, we describe our contributions in more detail. For simplicity of exposition, in this section we will assume that the mixing weights are uniform, i.e. $p_{i}=1 / k$ for all $i \in[k]$, although as we show, our algorithms can handle non-uniform mixing weights.

Our main results for learning MLRs are twofold. First, in the well-studied case where there is no regression noise, we show:

Theorem 1.1 (Informal). Assume that noise rate $\varsigma=0$. Let $w_{1}, \ldots, w_{k} \in \mathbb{R}^{d}$ be parameters of an unknown MLR $\mathcal{D}$ with separation $\Delta$. There is an algorithm which takes $N=\widetilde{O}(d) \cdot \exp (\widetilde{O}(\sqrt{k}))$ samples $^{1}$ from $\mathcal{D}$, runs in time $\widetilde{O}(N \cdot d)$, and outputs $\widetilde{w}_{1}, \ldots, \widetilde{w}_{k} \in \mathbb{R}^{d}$ so that with high probability, there exists some permutation $\pi$ satisfying $\left\|w_{i}-\widetilde{w}_{\pi(i)}\right\|_{2} \leq \frac{\Delta}{k^{100}}$ for all $i \in[k]$.

\footnotetext{
${ }^{1}$ Throughout the paper we let $\widetilde{O}(f)=O\left(f \log ^{c}(f)\right)$ for some universal constant $c$.
}

By combining this "warm start" with the boosting result of [30], we can also obtain arbitrary accuracy with minimal overhead in sample complexity and runtime.

In the case when noise rate $\varsigma$ is large, we obtain a similar result, though with an additional exponential dependence on $\Delta$ :

Theorem 1.2 (Informal). Let $w_{1}, \ldots, w_{k} \in \mathbb{R}^{d}$ be parameters of an unknown $M L R \mathcal{D}$ with separation $\Delta$, and noise $\varsigma>0$. There is an algorithm which takes $N=\widetilde{O}(d) \cdot \exp \left(\widetilde{O}\left(\sqrt{k} / \Delta^{2}\right)\right)$ samples from $\mathcal{D}$, runs in time $\widetilde{O}(N \cdot d)$, and outputs $\widehat{w}_{1}, \ldots, \widehat{w}_{k} \in \mathbb{R}^{d}$ so that with high probability, there exists some permutation $\pi$ satisfying $\left\|w_{i}-\widehat{w}_{\pi(i)}\right\|_{2} \leq \frac{\Delta}{k^{100}}+O(\varsigma)$ for all $i \in[k]$.

In particular, if $\Delta=\Omega(1)$, we again attain runtime which is subexponential in $k$. In the special case when the mixing weights are all known, and assuming that $\varsigma=O\left(\frac{\Delta}{k^{2} \operatorname{polylog}(k)}\right)$, by combining this result with the local convergence result of [28], we can again attain arbitrarily good accuracy by slightly increasing the runtime; see the full version for details.

Finally, for the problem of learning mixtures of hyperplanes, we are able to obtain qualitatively similar results. Again, for simplicity of exposition, we assume the mixing weights are uniform just in the current section. We obtain:

Theorem 1.3 (Informal). Let $\epsilon>0$, and let $v_{1}, \ldots, v_{k} \in \mathbb{R}^{d}$ be parameters of a mixture of hyperplanes $\mathcal{D}$ with separation $\Delta>0$. There is an algorithm which takes $N=\widetilde{O}(d) \cdot \exp \left(\widetilde{O}\left(k^{0.6}\right)\right)$ samples from $\mathcal{D}$, runs in time $\widetilde{O}(N \cdot d)$, and which outputs $\widehat{v}_{1}, \ldots, \widehat{v}_{k} \in$ $\mathbb{R}^{d}$ so that with high probability, there is a permutation $\pi$ so that $\left\|v_{i}-\widehat{v}_{\pi(i)}\right\|_{2} \leq \frac{\Delta}{k^{100}}$ for all $i \in[k]$.

\subsection{Related Work}

Mixtures of linear regressions were introduced in [11], and later by [23], under the name of hierarchical mixtures of experts, and have been studied extensively in the theory and ML communities ever since. Previous work on the problem with provable guarantees can roughly speaking be divided into three groups. Some of the previous work focuses on special cases of the problem, in particular, when the number of components is small [3, 10, 27, 29]. In contrast, we focus on the setting where $k$ is quite large, which is the setting which is typically true in applications, but is also much more algorithmically complicated.

Another line of work has focused on demonstrating local convergence guarantees for non-convex methods such as expectation maximization or alternating minimization [3, 17, 27-30, 46-48]. These papers demonstrate that given a sufficiently good warm start, non-convex methods are able to boost this warm start to arbitrarily good accuracy. These results should be viewed as largely complementary to our results, as our main result is a method which is able to provably achieve a good warm start. That said, we also demonstrate new algorithms for learning given a warm start that work under a weaker initialization and can tolerate more regression noise than was previously known in the literature.

The final class of results use moment-based methods to learn MLRs. Here, the literature has focused largely on the case of $\varsigma=0$ and spherical covariates, that is, covariates all drawn from $\mathcal{N}(0, \mathrm{I}){ }^{2}$

${ }^{2}$ To the best of our knowledge, the primary exception to this is [30], which considered noise-less MLRs whose components' covariates are drawn from arbitrary unknown 
A line of work has studied tensor decomposition-based methods [6, $40,47,48]$. However, these require additional non-degeneracy conditions on the MLR instance beyond separation. Indeed, as we argued in the Introduction, and more formally in the full version, moment based methods cannot obtain runtime which is sub-exponential in $k$. The work that closest to ours, and that we build off of, is that of [30], which demonstrates an algorithm which runs in $2^{\widetilde{O}(k)}$ for learning a MLR under separation conditions. However, as their algorithm is ultimately moment based, since it interacts through the samples through the moment-based univariate GMM learning algorithm of [34], it cannot achieve runtime sub-exponential in $k$.

List-Decodable Regression. A related problem to-indeed, a generalization of-the problem of learning MLRs is that of list-decodable regression $[8,26,39]$. Here, we assume that we are given a set of data points $\left(x_{1}, y_{1}\right), \ldots,\left(x_{n}, y_{n}\right)$, where an $\alpha$-fraction of them come from an unknown linear regression $y_{i}=\left\langle w, x_{i}\right\rangle+\eta$, where $x_{i}$ is Gaussian, $\eta$ is Gaussian noise, and $\alpha<1 / 2$. The goal is then to recover a list of $O(1 / \alpha)$ possible $w_{1}, \ldots, w_{O(1 / \alpha)} \in \mathbb{R}^{d}$ so that $\left\|w_{i}-w\right\|_{2}$ is small for at least one element in the list.

It is not hard to see that given a uniform MLR instance, if we feed it into an algorithm for list-decodable regression, the list must contain something which is close to each of the regressors in the MLR instance, as each mixture component is an equally valid solution to the list-decodable regression problem. Thus one could hope that these algorithms for list-decodable regression could yield improved algorithms for learning MLRs as well.

Unfortunately, all known techniques, including the state of the art [26, 39], either are too weak to be applied to our setting, or use the Sum-of-Squares SDP hierarchy and again interact through the data via estimating high-degree moments of the distribution. As a result, these latter algorithms still suffer runtimes which are exponential in $k$.

Subspace Clustering. The mixtures of hyperplanes problem we consider in this paper can be thought of as a special case of the subspace clustering or hyperplane clustering problem, where data is thought of as being drawn from a union of linear subspaces. In our problem, we additionally assume that the data is Gaussian within each subspace. The literature on subspace clusterings is vast and we cannot do it justice here; see [16, 37, 43] and references therein for a more complete treatment. On the one hand, the mixture of hyperplanes problem arises naturally in practical contexts of projective motion segmentation [44] and hybrid system identification [2]. On the other, it also corresponds to a challenging setting of the problem due to the low codimensionality of the subspaces. Indeed, essentially all algorithms for subspace clustering with provable guarantees either run in time exponential in the dimension of the subspaces (e.g. RANSAC [18], algebraic subspace clustering [45], spectral curvature clustering [31]) or require the codimension to be at least some small but constant fraction of the ambient dimension $[5,16,32,41]$. To our knowledge the only work which addresses the codimension 1 case is [42], though their setting and guarantees are quite different from ours.

Gaussians satisfying some condition number bounds and obtained a $d \cdot \exp \left(k^{2}\right)$ algorithm in this setting.
The Fourier Transform in Distribution Learning. One of our main algorithmic tools will be the univariate (continuous) Fourier transform, as a way to estimate Fourier moments of our distribution. In recent years, the question of learning the Fourier transform of a function has attracted a considerable amount of interest in theoretical computer science [9, 20, 22, 24, 25, 33, 35, 38]. Our application is somewhat different in that we have explicit access to the function we will take the Fourier transform of.

In the context of distribution learning, the discrete Fourier transform has been used to learn families of distributions such as sums of independent integer random variables [14], Poisson Binomial distributions [15], and Poisson multinomial distributions [13, 14]. These algorithms typically work by exploiting Fourier sparsity of the underlying distribution. However, the way we use the Fourier transform is quite different: we only use it to compute different statistics of the data, namely, the Fourier moments of our distribution.

Univariate Density Estimation. Another important algorithmic primitive we use is univariate density estimation and specifically, the piecewise polynomial-based estimators given in [1, 7]. Univariate density estimation has a long history in statistics, ML, and theoretical computer science, and a full literature review of the field is out of the scope of this paper; see e.g. [12] for a more comprehensive overview of the literature. However, to the best of our knowledge, there are few previous cases where univariate density estimation has been used as a key tool for a high dimensional learning task.

\section{PRELIMINARIES}

\subsection{Probabilistic Models}

In this section, we formally define the models we consider throughout this paper, namely, mixtures of linear regression and hyperplanes, and some important parameters for these models.

Definition 2.1 (Mixtures of Linear Regressions). Given mixing weights $p \in[0,1]^{k}$ with $\sum_{i=1}^{k} p_{i}=1$, regressors $w_{1}, \cdots, w_{k} \in$ $\mathbb{R}^{d}$, and noise rate $\varsigma \geq 0$, the corresponding mixture of spherical linear regressions (or simply mixture of linear regressions) is the distribution over pairs $(x, y) \in \mathbb{R}^{d} \times \mathbb{R}$ where $x \sim \mathcal{N}(0, \mathrm{I})$ and $y=\left\langle w_{i}, x\right\rangle+g$ for $w_{i}$ sampled with probability $p_{i}$ and $g \sim \mathcal{N}\left(0, \varsigma^{2}\right)$.

When $\varsigma=0$, we say that the MLR is noiseless.

In this paper, we will study the parameter learning problem for mixtures of linear regressions, that is, we wish to recover the parameters of the mixture. To this end, we will need some assumptions to ensure that the regressors are uniquely identifiable. These assumptions are standard throughout the literature. Given a mixture of linear regressions $\mathcal{D}$, let $\Delta=\min _{i \neq j}\left\|w_{i}-w_{j}\right\|_{2}$ be the minimum $L_{2}$ separation among all $w_{i}$ in the mixture, and we let $p_{\text {min }}=\min _{i \in[k]} p_{i}$. To normalize the instance, we will also assume that $\left\|w_{i}\right\|_{2} \leq 1$ for all $i=1, \ldots, k$. However, more generally, our algorithms will have a mild polynomial dependence on the maximum $L_{2}$ norm of any $w_{i}$. We omit this case for simplicity of exposition.

Definition 2.2. Given mixing weights $p \in[0,1]^{k}$ with $\sum_{i=1}^{k} p_{i}=1$ and unit vectors $v_{1}, \cdots, v_{k} \in \mathbb{S}^{d-1}$, the corresponding mixture of hyperplanes is the distribution with law given by $\sum_{i=1}^{k} p_{i} \mathcal{N}\left(0, \Pi_{i}\right)$ where $\Pi_{i} \triangleq \mathbf{I}-v_{i} v_{i}^{\top} \in \mathbb{R}^{d \times d}$. 
As before, we need some assumptions on the parameters to ensure identifiability. Like before, let $p_{\text {min }}=\min _{i \in[k]} p_{i}$. Because $v_{i}$ are now only identifiable up to sign, we define $\Delta$ to be the minimum quantity such that or all $i \neq j$ and $\epsilon_{i}, \epsilon_{j} \in\{ \pm 1\},\left\|\epsilon_{i} v_{i}-\epsilon_{j} v_{j}\right\|_{2} \geq \Delta$.

\subsection{Miscellaneous Notation}

For real-valued functions $\mathcal{F}: \mathbb{R} \rightarrow \mathbb{R}$ and $p \in \mathbb{N}$, we will use $\mathcal{M}_{p}(\mathcal{F})$ to denote $\int_{-\infty}^{\infty} x^{p} \cdot \mathcal{F}(x) \mathrm{d} x$. Given $f \in L^{2}(\mathbb{R})$, the Fourier transform of $f$ is denoted by $\widehat{f}[\omega]=\int_{-\infty}^{\infty} f(x) \cdot e^{-2 \pi \mathrm{i} \omega x} \mathrm{~d} x$. We will denote by $\Delta^{n}$ the probability simplex of vectors in $\mathbb{R}^{n}$ with nonnegative entries summing to 1 . We will occasionally use notation like $x=\left[c_{1}, c_{2}\right] \cdot y$ and $x=1 \pm \delta$ to mean $c_{1} y \leq x \leq c_{2} y$ and $1-\delta \leq x \leq 1+\delta$ respectively. We will sometimes refer to a univariate mixture $\mathcal{F}$ of zero-mean Gaussians with mixing weights $p \in \Delta^{k}$ and variances $\sigma_{1}^{2}, \ldots, \sigma_{k}^{2}$ as a mixture of $k$ univariate zeromean Gaussians "with parameters $\left(\left\{p_{i}\right\}_{i \in[k]},\left\{\sigma_{i}\right\}_{i \in[k]}\right)$." We will define $\sigma_{\min }(\mathcal{F}) \triangleq \min _{i \in[k]} \sigma_{i}$ and $\sigma_{\max }(\mathcal{F}) \triangleq \max _{i \in[k]} \sigma_{i}$ and refer to $\sigma_{\min }(\mathcal{F})^{2}$ and $\sigma_{\max }(\mathcal{F})^{2}$ as the minimum and maximum variance of $\mathcal{F}$, respectively.

\section{OVERVIEW OF TECHNIQUES}

Here we give a high-level overview of our algorithms. For clarity of exposition, in this section we will assume $p_{\min }=1 / k$ and $\Delta=\Theta(1)$.

\subsection{Fourier Moment Descent}

We first describe our techniques that achieve Theorem 1.1, before describing how to adapt them to achieve Theorems 1.2 and 1.3.

We begin by briefly recapping the moment descent algorithm of [30]. Moment descent is an iterative algorithm which attempts to find the parameters of one component at a time as follows. Let $w_{1}, \ldots, w_{k} \in \mathbb{R}^{d}$ be the parameters of a MLR $\mathcal{D}$ with separation $\Delta>0$ and noise rate $\varsigma=0$, and again for simplicity let us assume that the mixing weights are uniform. To learn a single regressor, the idea is to maintain a guess $a_{t} \in \mathbb{R}^{d}$ for one of the regressors at each time step $t$, and iteratively refine it by making random steps and checking progress. The progress measure they consider is simply $\sigma_{t}^{2} \triangleq \min _{i \in[k]}\left\|w_{i}-a_{t}\right\|_{2}^{2}$.

Concretely, the algorithm proceeds as follows. By a straightforward PCA step, we can essentially assume that $d \leq k$. Given a guess $a_{t}$, we update by sampling a random unit vector $z \in \mathbb{S}^{d-1}$, defining $a_{t+1}^{\prime} \triangleq a_{t}-\eta_{t} \cdot z \in \mathbb{R}^{d}$ for some learning rate $\eta_{t}$, and letting $\left(\sigma_{t}^{\prime}\right)^{2} \triangleq \min _{i \in[k]}\left\|w_{i}-a_{t}\right\|_{2}^{2}$. In general, for $a \in \mathbb{R}^{d}$, the univariate distribution of the residual $y-\langle a, x\rangle$, where $(x, y) \in \mathbb{R}^{d} \times \mathbb{R}$ is sampled from $\mathcal{D}$, is distributed as $\frac{1}{k} \sum_{i=1}^{k} \mathcal{N}\left(\mu,\left\|w_{i}-a\right\|_{2}^{2}\right)$, that is, it is distributed as a mixture of univariate Gaussians with mixing weights which are the same as those of $\mathcal{D}$, and variances which are equal to the squared $L_{2}$ distances between the regressors and a. In particular, to estimate $\min _{i \in[k]}\left\|w_{i}-a_{t}\right\|_{2}^{2}$, one can learn the univariate mixture sufficiently well via [34], and simply read off the minimum variance. By doing so, one can check if $\sigma_{t}^{\prime}<\sigma_{t}$. If so, we set $a_{t+1}=a_{t+1}^{\prime} \in \mathbb{R}^{d}$, and repeat.

The main bottleneck in this routine is the univariate learning step. Specifically, the algorithm of [34] relies on the method of moments to learn the parameters of the univariate mixture of Gaussians, and as a result, takes $k^{O(k)}$ samples and time. In fact, this is inherent: [34] demonstrates that $k^{\Omega(k)}$ samples are necessary to learn the parameters of a mixture of Gaussians, precisely by leveraging moment matching instances.

However, all we need is an estimate of the minimum variance of the mixture of Gaussians. One can first observe that it is possible to estimate the maximum variance of a component in a univariate mixture of Gaussians based on a sufficiently high degree moment. This is because the $p$-th moment of a uniform mixture of Gaussians $\mathcal{F}$ with variances $s_{1}^{2}, \ldots, s_{k}^{2}$ has the following form, for $p$ even:

$$
\underset{Z \sim \mathcal{F}}{\mathbb{E}}\left[Z^{p}\right]=\sum_{i=1}^{k} \frac{1}{k} \cdot s_{i}^{p} \cdot(p-1) ! !=[c / k, 1] \cdot \sigma_{\max }(\mathcal{F})^{p} \cdot p^{p / 2},
$$

for $\sigma_{\max }(\mathcal{F}) \triangleq \max _{i \in[k]} s_{i}$ and some universal constant $c>0$. Therefore, for any $\kappa>0$, if we set $p=\Theta(\log k / \log (1+\kappa))=$ $\Theta\left(\kappa^{-1} \log k\right)$, we have that $p^{-1 / 2} \mathbb{E}_{Z \sim \mathcal{F}}\left[Z^{p}\right]^{1 / p}$ is a $(1-\Theta(\kappa))$ approximation of $\sigma_{\max }(\mathcal{F})$. Moreover, we can estimate this in $p^{O(p)}=2^{\widetilde{O}\left(\kappa^{-1} \log k\right)}$ samples by standard concentration arguments which we defer to the full version.

Unfortunately, a priori this argument says nothing about estimating the minimum variance. It is easy to see that two mixtures of univariate zero-mean Gaussians $D_{1}, D_{2}$ can have very similar $p$-th moments but wildly different minimum variances (e.g. take $D_{1}$ to be a single Gaussian $\mathcal{N}\left(0, k^{100}\right)$, and take $D_{2}$ to be a uniform mixture of $\mathcal{N}\left(0, k^{100}\right)$ and $\left.\mathcal{N}(0,1)\right)$.

The key insight is that while higher-degree moments of a mixture $D$ of zero-mean Gaussians tell us nothing about the minimum variance, those of its Fourier transform do:

OBSERVATION 3.1. If the components of $D$ have variances $\left\{s_{i}^{2}\right\}$ and mixing weights $\left\{p_{i}\right\}$, the Fourier transform of the density of $D$ is a new (unnormalized) mixture of Gaussians with variances $\left\{\Theta\left(s_{i}^{-2}\right)\right\}$ and mixing weights proportional to $\left\{p_{i} / s_{i}\right\}$.

In particular, if we have a sufficiently good estimate of the maximum variance of any component in the Fourier transform of $D$, then by inverting this estimate, we can estimate the minimum variance of any component in $D$. So if we had access to the Fourier transform of $D$, we could use the moments of this distribution to estimate the maximum variance of any component of the Fourier transform and thus learn the minimum variance of $D$.

What remains is to estimate moments of the Fourier transform of $D$ using solely samples from $D$. Here we use existing primitives for univariate density estimation $[1,7]$ to obtain an explicit approximation $\widetilde{D}$ to the density of $D$, after which we can explicitly compute moments of the Fourier transform of $\widetilde{D}$. We defer the technical details of how to argue that its moments are close to those of the Fourier transform of $D$ to Section 5.1, as they are rather involved. In short, this allows us to achieve the same sorts of guarantees for estimating min-variance as for estimating max-variance: for any $\kappa>0$, we can learn the minimum variance of the mixture to multiplicative error $1+O(\kappa)$ with $2^{\widetilde{O}\left(\kappa^{-1} \log k\right)}$ samples and time.

However, there is an important subtlety here. Namely, the quality of the approximation to the minimum variance we can obtain strongly depends on the degree of the Fourier moment we use. The degree in turn dictates the sample complexity of the algorithm: the higher the Fourier degree we need, the better the univariate density estimate must be, and therefore, the more samples we need 
in order to adequately perform the density estimate. Therefore, if the difference between $\sigma_{t}$ and $\sigma_{t}^{\prime}$ is too small, we cannot reliably check if we've made progress without taking too many samples. Unfortunately, the difference between these two quantities is typically quite small. Since $a_{t+1}^{\prime}$ is a random perturbation of $a_{t}$, we have that with probability $1 / \operatorname{poly}(k)$, it holds that $\sigma_{t}^{\prime} \leq(1-\Omega(1 / k)) \sigma_{t}$, and moreover, this is tight. In particular, this says that we would need to take $\kappa=\Omega(1 / k)$ in the discussion above, which would result in a $2^{\Theta(k)}$ runtime, which we wish to avoid.

However, we show that with subexponentially large probability, the difference is sufficiently large so that we can detect this difference using subexponentially many samples. In particular, observe that for any constant $0<c<1 / 2$, if $z$ is a random unit vector in the span of $\left\{w_{i}-a_{t}\right\}$, then with probability $\exp \left(-k^{1-2 c}\right)$,

$$
\left\langle z, \frac{w_{i}-a_{t}}{\left\|w_{i}-a_{t}\right\|_{2}}\right\rangle \geq \Omega\left(k^{-c}\right),
$$

in which case if we define $a_{(t+1)}^{\prime}$ as previously, we get that with probability $\exp \left(-k^{1-2 c}\right), \sigma_{t}^{\prime} \leq\left(1-\Omega\left(\frac{1}{k^{2 c}}\right)\right) \sigma_{t}$. So by trying superpolynomially many random directions $z$ at every step, we ensure with high probability that one of those directions will make $1-$ $\Omega\left(k^{-2 c}\right)$ progress, for some $c<1$. By combining this with our certification procedure described above, we can make non-trivial progress in the algorithm with sub-exponentially many samples.

By iterating this update, we can obtain $a_{T}$ so that $\left\|a_{T}-w_{i}\right\|_{2} \leq$ $\frac{\Delta}{k^{100}}$ in subexponential time. We call this subroutine Fourier moment descent, and it allows us to learn a single regressor to good accuracy. For technical reasons, the complexity of this approach grows as we get closer to $w_{i}$, however, it allows us to obtain a very good "warm start". In the noiseless case, this can be combined with the boosting procedure from [30] to obtain arbitrary accuracy.

This technology allows us to learn a single regressor to very high accuracy. In the noiseless setting, this lets us "peel off" the samples from this component almost completely, and we can now repeat on the sub-mixture with this component removed to learn another component, and iterate to eventually learn all the regressors.

That said, as we shall see, this is much trickier with noise.

\subsection{Learning with Regression Noise}

What changes when we assume that there is a significant amount of noise $\varsigma$ ? From the perspective of our Fourier moment descent algorithm, it turns out not much does, at least to a certain extent: in fact, essentially the same argument goes through and allows us to learn a single component to error at most $\left\|a_{T}-w_{i}\right\|_{2} \leq \frac{\Delta}{k^{100}}+O(\varsigma)$ where $\varsigma$ is the standard deviation of the white noise.

However, learning all components becomes substantially more difficult. In particular, the peeling process no longer works: the regression noise precludes perfectly removing the influence of a component that we have learned from the rest of the mixture.

To avoid this, we circumvent the need for peeling altogether. By a delicate analysis, we will show that with decent probability, we can control the dynamics of the Fourier moment descent algorithm, so that it will converge to the regressor that it was initially closest to. This is the key technical ingredient behind getting Fourier moment descent to handle regression noise.
We sketch its proof below. Let $a_{t}$ be the current iterate of Fourier moment descent, and suppose that $i^{*}=\operatorname{argmin}_{i \in[k]}\left\|w_{i}-a_{t}\right\|_{2}$. Then, one can show that if $a_{t+1}^{\prime} \in \mathbb{R}^{d}$ is a random perturbation of $a_{t}$, then with probability at least $\exp \left(-\Omega\left(1 / \Delta^{2}\right)\right) / \operatorname{poly}(k)$, we have that $i^{*}$ is still the closest component to $a_{t+1}^{\prime}$. Moreover, this bound is tight up to polynomial factors, if all we assume about the $w_{i}$ is that they are $\Delta$-separated. One could hope that by using this sort of argument, we could argue that with at least subexponentially large probability, we always stay closest to $w_{i^{*}}$. However, this argument runs into a couple of difficulties, which we address one at a time.

The first difficulty is that while this happens with decent probability for any individual $a_{t+1}^{\prime} \in \mathbb{R}^{d}$, our algorithm will typically need to try sub-exponentially many perturbations before we find one that makes progress. Thus the naive union bound over all sub-exponentially many $a_{t+1}^{\prime} \in \mathbb{R}^{d}$ would be far too loose to say anything here. To get around this, we instead demonstrate that conditioning on the event that $\sigma_{t}^{\prime}<\sigma_{t}$, we remain closest to $i^{*}$ with non-trivial probability.

However, even this is insufficient, as we only have a multiplicative estimate of $\sigma_{t}$ and $\sigma_{t}^{\prime}$. To get around this, we make a stronger assumption: we assume that not only is our iterate the closest to $w_{i^{*}} \in \mathbb{R}^{d}$, but we also assume that its distance to the other $w_{i} \in \mathbb{R}^{d}$ for $i \neq i^{*}$ is at least some multiplicative factor larger than its distance to $w_{i^{*}} \in \mathbb{R}^{d}$. Specifically, we assume that

$$
\left\|w_{i}-a_{t}\right\|_{2} \geq\left(1+c \frac{\Delta^{2}}{\sqrt{k}}\right)\left\|w_{i^{*}}-a_{t}\right\|_{2},
$$

for all $i \neq i^{*}$, and some constant $c>0$. By making this stronger assumption, we are able to demonstrate that with probability at least $1 / \operatorname{poly}(k)$, this gap is maintained for the $a_{t+1}^{\prime}$ which Fourier moment descent chooses as the next iterate.

Our overall algorithm then will be to demonstrate an initialization scheme for $a_{0}$ so that for any $i^{*} \in[k]$, (2) holds for $a_{0}$ and that $i^{*}$ with probability at least $\exp \left(-\widetilde{O}\left(\sqrt{k} / \Delta^{2}\right)\right)$. If we can do so, then if we run Fourier moment descent starting from these random initializations enough times, then eventually with high probability we will output multiple estimates for each $w_{i}$ for $i=1, \ldots, k$, and then we can simply run a basic clustering algorithm to recover all of the regressors. Furthermore, because each run of moment descent only needs to go on for $\widetilde{O}\left(\sqrt{k} / \Delta^{2}\right)$ iterations, and at each iteration we stay closest to the same component that the previous iterate was closest to with $1 / \operatorname{poly}(k)$ probability, it suffices to try $\exp \left(\widetilde{O}\left(\sqrt{k} / \Delta^{2}\right)\right)$ random initializations for all this to work.

Lastly, it turns out this initialization scheme is also quite delicate. For instance, if we simply chose random initializations over the unit ball, then these will, with overwhelming probability, favor being close to $w_{i}$ with small norm. If we have regressors with different norms, we will thus likely never be close to a $w_{i} \in \mathbb{R}^{d}$ with large norm in our initializations, and as a result, cannot argue that our Fourier moment descent algorithm will ever recover this regressor. To get around this, we demonstrate a gridding scheme, where we initialize randomly over spheres of up to radius $r$, for $r$ on a fine grid between 0 and $O\left(k^{1 / 4}\right)$.

We emphasize that this is quite counterintuitive: when we start at radius $O\left(k^{1 / 4}\right)$, we are exceedingly far away from every $w_{i} \in \mathbb{R}^{d}$, as they are assumed to have norm at most 1 . However, our analysis 
of Fourier moment descent works fine in this setting, and by having such large radius, we are able to ensure that for each $i^{*} \in[k],(2)$ holds for $a_{0}$ and $i^{*}$ with at least the desired probability. The details are quite technically involved, and we defer them to Section 6.

More Noise-Robust Boosting. As mentioned previously, in the noiseless setting, the boosting algorithm of [30] allows us to bootstrap our warm start, obtained via Fourier moment descent, to arbitrarily high accuracy. It turns out that in the noisy setting, their boosting algorithm also allows one to go slightly below $O(\varsigma)$. Interestingly, motivated again by the connection to Fourier analysis, we demonstrate an improved boosting algorithm that is able to tolerate substantially more noise as well as a much weaker warm start.

The boosting algorithm of [30] is based on SGD on a regularized form of gravitational potential, which was notably used in [21]. While this objective is concave, they demonstrate that in a small neighborhood around the true regressors, SGD updates based on this objective contract in expectation and hence make progress.

In contrast, we propose an update based on a regularized form of the cosine integral objective $g(v) \triangleq \mathbb{E}_{(x, y) \sim \mathcal{D}} \mathrm{Ci}(|\langle v, x\rangle-y|)$. This objective looks much worse behaved: it is neither convex nor concave-indeed, it is not even monotone! However, the key technical fact which makes this objective more noise tolerant is precisely Observation 3.1. This allows us to argue that the contribution to the gradient of the objective from the "good" component, which we are close to, dominates the contribution of the gradient from the other "bad" components. At a high level, ignoring many technical issues for the time being, this is because if $v$ is the current iterate, a main part of the contribution to the gradient from component $i$ is $\mathbb{E}_{g \sim \mathcal{N}\left(0, \beta_{i}^{2}\right)}[\cos (g)]$, where $\beta_{i}$ is a monotone function of $\left\|w_{i}-v\right\|_{2}$. This is exactly the real part of the Fourier transform of $\mathcal{N}\left(0, \beta_{i}^{2}\right)$, and the fact that this decreases as $\exp \left(O\left(\beta_{i}^{-2}\right)\right)$ follows precisely from Observation 3.1. This allows us to have much finer control over the contribution from the "bad" components, which allows us to tolerate significantly more noise and a substantially weaker warm start. For the quantitative details, see the full version.

\subsection{Learning Mixtures of Hyperplanes}

Mixtures of hyperplanes are similar enough to MLRs that an appropriate instantiation of our techniques also suffices for this problem.

It is not hard to show that vanilla moment descent can be modified as follows to get a $\widetilde{O}(d \cdot \exp (\widetilde{O}(k)))$-time algorithm for mixtures of hyperplanes. First it is not hard to see that as with spherical MLRs, here we can still effectively reduce the dimension of the problem from $d$ to $k$. At time $t$ we still maintain an estimate $a_{t}$ for one of the components, and in lieu of the usual progress measure $\min _{i \in[k]}\left\|w_{i}-a_{t}\right\|_{2}$ to which we no longer have access, we can use the modified progress measure $\sigma_{t} \triangleq \min _{i \in[k]}\left\|\Pi_{i} a_{t}\right\|_{2}$ (See Definition 2.2 for definition of $\Pi_{i} \in \mathbb{R}^{d \times d}$ ) We can estimate $\sigma_{t}$ in $\exp (\widetilde{O}(k))$ time by simply projecting in the direction of $a_{t}$ itself to get a mixture of univariate Gaussians with variances $\left\{\left\|\Pi_{i} a_{t}\right\|_{2}^{2}\right\}_{i \in[k]}$ and learning that mixture via [34]. This leads to the following straightforward modification of moment descent: repeatedly update $a_{t} \in \mathbb{R}^{d}$ by sampling many random steps $\left\{\eta_{t} \cdot z_{j}\right\}$ for step size $\eta_{t}$ and $z_{j} \in \mathbb{S}^{d-1}$ and taking one of them to get to $a_{t+1} \in \mathbb{R}^{d}$ if it contracts the progress measure.
One immediate issue with this approach, even for achieving $\widetilde{O}(d) \cdot \exp (\widetilde{O}(k))$ runtime, is that if we don't control $\left\|a_{t}\right\|_{2}$, then for all we know $\sigma_{t}^{2}$ contracts simply because our random steps are taking us closer and closer to 0 . The natural workaround is to insist $a_{t} \in \mathbb{S}^{d-1}$ for all $t$ by projecting onto $\mathbb{S}^{d-1}$ after every step. That is, in every iteration of moment descent, given a random collection of candidate steps $\left\{\eta \cdot z_{j}\right\}_{j}$, choose one for which, if we define $a_{t+1}=\frac{a_{t}+\eta \cdot z_{j}}{\left\|a_{t}+\eta \cdot z_{j}\right\|_{2}}$, then the progress measure contracts. One can show this already suffices to get a $\widetilde{O}(d) \cdot \exp (\widetilde{O}(k))$-time algorithm for learning mixtures of hyperplanes.

The extra projection onto $\mathbb{S}^{d-1}$ at every step introduces a variety of technical challenges for trying to implement the strategy of Section 3.1 to achieve sub-exponential runtime. Specifically, in order to carry out the balancing of parameters from Section 3.1, one needs to be careful in choosing the right events, analogous to the event in (1), such that 1) conditioned on those events $\sigma_{t}$ contracts by at least a factor of $1-\Omega\left(k^{-a}\right)$ for some $a>0$, and 2) these events all occur with probability $\exp \left(-k^{1-a^{\prime}}\right)$ for some $a^{\prime}>0$.

If for instance $a_{t}$ is positively correlated with some $v_{i^{*}} \in \mathbb{R}^{d}$, it turns out the right events to choose are that the random step is both $k^{-1 / 5}$-positively correlated with $\frac{\Pi_{i^{*}} a_{t}}{\left\|\Pi_{i^{*}} a_{t}\right\|_{2}}$ and at least $k^{-1 / 5}$ negatively correlated with $v_{i^{*}} \in \mathbb{R}^{d}$, and because these directions are orthogonal, if $z_{j}$ is a random vector in the span of $v_{1}, \ldots, v_{k}$ then we could lower bound the probability of both events occurring by the product of the probabilities they individually occur, which is $\exp \left(-k^{3 / 5}\right)$, and get 2) for $a^{\prime}=2 / 5$. The analysis for showing 1 ) (for $a=3 / 5)$ is involved, so we defer it to Section 7.1. These together yield a $\widetilde{O}(d) \cdot \exp \left(k^{3 / 5}\right)$-time algorithm to learning one component of a mixture of hyperplanes.

To learn all components, we would like to implement some kind of boosting procedure. Our approach here is to regard $\mathcal{D}$ in a certain way as a non-spherical MLR with well-conditioned covariances, at which point we can invoke, e.g., the boosting algorithm of [30]. We defer these details to the full version. Once we are able to refine an estimate for a direction of $\mathcal{D}$ to arbitrary precision, we can carry out the "peeling" procedure outlined at the end of Section 3.1 to learn all components.

\section{ROADMAP}

In Section 5 we present our Fourier moment descent algorithm for learning a single component. In Section 6 we demonstrate a modification of our algorithm to learn all the components in the presence of regression noise. In Section 7 we demonstrate our subexponential time algorithm for learning a mixture of hyperplanes.

\section{WARM START VIA FOURIER MOMENT DESCENT}

Here we propose a technique for moment descent based on approximating the minimum variance of a component in a mixture of univariate zero-mean Gaussians. The main result of this section is an algorithm, which we call FouRIERMOMENTDESCENT, for learning a single component of a mixture of $k$ linear regressions in time and sample complexity sub-exponential in $k$ : 
THeOREM 5.1. Given $\delta, \epsilon>0$ and a mixture of spherical linear regressions $\mathcal{D}$ with separation $\Delta$ and noise rate $\varsigma=O(\epsilon)$, there is an algorithm (FOURIERMOMENTDESC ENT $(\mathcal{D}, \delta, \epsilon)$ inAlgorithm 4) that outputs a vector $v \in \mathbb{R}^{d}$ such that with probability $1-\delta$, we have $\left\|w_{i}-v\right\|_{2} \leq O(\epsilon)$ for some $i \in[k]$. Furthermore, FoURIERMOMENTDESCENT requires sample complexity

$N=\widetilde{O}\left(d \epsilon^{-2} \ln (1 / \delta) p_{\min }^{-4}\right.$ poly $\left.\left(k, \ln \left(1 / p_{\min }\right), \ln (1 / \epsilon)\right)^{\sqrt{k} \ln \left(1 / p_{\min }\right)}\right)$ and time complexity $N d \cdot \operatorname{poly} \log \left(k, d, 1 / \Delta, 1 / p_{\min }, 1 / \epsilon\right)$.

In Section 5.1 we give an algorithm for estimating the minimum variance of a mixture of univariate, zero-mean Gaussians via its Fourier transform. In Section 5.2 we show how to leverage this technology to obtain our algorithm FoURIERMOMENTDESCENT.

\subsection{Estimating Minimum Variance}

Here we give the key primitive underlying all of the algorithmic results of this work: an algorithm for estimating the minimum variance of a mixture of zero-mean Gaussians. This requires some setup regarding existing technology for density estimation.

5.1.1 Density Estimation in $L_{2}$. Our main density estimation tool will be piecewise polynomials. We favor them because there are clean algorithms for density estimation via piecewise polynomials, and the form of the estimator will be useful for us later on. Formally:

Definition 5.2. An $s$-piecewise degree- $d$ polynomial $p: \mathbb{R} \rightarrow \mathbb{R}$ is specified by a collection of intervals $I_{1}=\left[-\infty, a_{1}\right], I_{2}=\left[a_{1}, a_{2}\right], I_{3}=$ $\left[a_{2}, a_{3}\right], \ldots, I_{s-1}=\left[a_{s-2}, a_{s-1}\right], I_{s}=\left[a_{s-1},+\infty\right]$ and $s$ degree- $d$ polynomials $p_{1}, \ldots, p_{s}$ such that for any $i \in[s]$ and $x \in I_{s}, p(x)=p_{i}(x)$. We refer to $a_{1}, \ldots, a_{s-1}$ as the nodes of $p$.

We will use the following algorithm as a black box:

Theorem 5.3 (Theorem 43 IN [1]). For any $\eta>0$, there is an algorithm that, given sample access to a mixture $\mathcal{F}$ of $k$ univariate Gaussians, outputs a $O(k)$-piecewise degree-O $(\log 1 / \eta)$ polynomial hypothesis distribution $\mathcal{F}^{\prime}$ for which $d_{\mathrm{TV}}\left(\mathcal{F}, \mathcal{F}^{\prime}\right) \leq \eta$, using $N=$ $O\left(\left(k / \eta^{2}\right) \ln (1 / \eta) \ln (1 / \delta)\right)$ samples and running in time $\widetilde{O}(N)$.

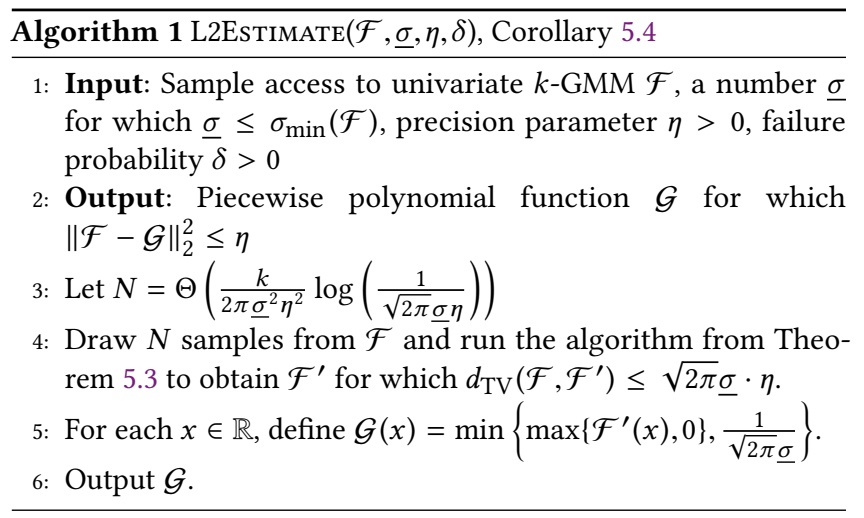

Corollary 5.4. For any $0<\eta, \delta<1$, mixture of $k$ univariate Gaussians $\mathcal{F}$, and $\underline{\sigma}>0$ for which $\underline{\sigma} \leq \sigma_{\min }(\mathcal{F})$, with probability at least $1-\delta$ L2EsTimate $(\mathcal{F}, \underline{\sigma}, \eta, \delta)$ (Algorithm 1) outputs a
$O(k \log (1 /(\eta \underline{\sigma})))$-piece degree-O $(\log (1 /(\eta \underline{\sigma}))$ polynomial hypothesis distribution $\mathcal{G}: \mathbb{R} \rightarrow \mathbb{R}_{\geq 0}$ for which $\|\mathcal{F}-\mathcal{G}\|_{2}^{2} \leq \eta$, using $N=O\left(\left(k / \eta^{2}\right) \log (1 /(\eta \underline{\sigma})) \log (1 / \delta)\right)$ samples and time $\widetilde{O}(N)$.

Proof. Because $\mathcal{F}$ has range in $\left[0, \frac{1}{\sqrt{2 \pi} \sigma_{\min }(\mathcal{F})}\right], d_{\mathrm{TV}}(\mathcal{F}, \mathcal{G}) \leq$ $d_{\mathrm{TV}}\left(\mathcal{F}, \mathcal{F}^{\prime}\right)$. By Holder's, it suffices to show $\|\mathcal{F}-\mathcal{G}\|_{\infty} \leq \frac{1}{\sqrt{2 \pi} \cdot \underline{\sigma}}$. Because $\mathcal{G}(x) \leq \mathcal{F}(x)$ for all $x \in \mathbb{R}$, it is enough to show $\|\mathcal{F}\|_{\infty} \leq$ $\frac{1}{\sqrt{2 \pi} \cdot \sigma}$, which holds because $\mathcal{F}$ is a convex combination of Gaussians of variance at least $\sigma_{\min }(\mathcal{F}) \geq \underline{\sigma}$. Note that $\mathcal{G}$ is a piecewise polynomial because we can refine the intervals defining $\mathcal{F}^{\prime}$ to incorporate the intersections of $\mathcal{F}^{\prime}$ with the lines $y=\frac{1}{\sqrt{2 \pi} \sigma}$ and $y=0$. Since each component can intersect with these lines at most $O(\log (1 /(\eta \underline{\sigma})))$ times since they have degree at most $O(\log (1 /(\eta \underline{\sigma})))$, this yields the desired bound on the number of pieces.

5.1.2 Minimum Variance Via Fourier Transform Moments. We now show how to use an $L_{2}$-close estimator for the density of a mixture $\mathcal{F}$ of zero-mean univariate Gaussians to approximate $\sigma_{\min }(\mathcal{F})$. First, we show how to estimate high moments of $\mathcal{F}$ :

LEMMA 5.5. For any even integer $p \in \mathbb{N}$ and $\xi>0$ the following holds. Let $\mathcal{F}: \mathbb{R} \rightarrow \mathbb{R}$ be an mixture of $k$ Gaussians given by $\mathcal{F}(x)=$ $\sum_{i=1}^{k} p_{i} \cdot \mathcal{N}\left(0, \sigma_{i}^{2} ; x\right)$, and define $L \triangleq \sum_{i=1}^{k} p_{i}$. Let $\bar{\sigma}>0$ be any number for which $\bar{\sigma} \geq \max _{i \in[k]} \sigma_{i}$.

Let $\tau=8 \bar{\sigma}^{2} \cdot \max (p, \ln (4 L / \xi))$ and $\eta=\frac{\xi^{2} p}{8 \tau^{2 p+1}}$. Then if $\mathcal{G}$ : $\mathbb{R} \rightarrow \mathbb{R}_{\geq 0}$ satisfies $\|\mathcal{F}-\mathcal{G}\|_{2}^{2} \leq \eta$, then $\mathcal{G}^{\prime}$ defined by $\mathcal{G}^{\prime}(x)=$ $\mathbb{1}[x \in[-\tau, \tau]] \cdot \mathcal{G}(x)$ for all $x \in \mathbb{R}$ satisfies $\left|\mathcal{M}_{p}(\mathcal{F})-\mathcal{M}_{p}\left(\mathcal{G}^{\prime}\right)\right| \leq \xi$.

Proof. For simplicity, we define $\sigma_{\max }=\sigma_{\max }(\mathcal{F})$. We would like to pick the truncation threshold $\tau$ so that $\int_{[-\tau, \tau]^{c}} x^{p} \cdot \mathcal{F}(x) \mathrm{d} x \leq$ $\xi / 2$. For this, it suffices to take $\tau$ for which

$$
x^{p} \leq e^{x^{2} /\left(4 \sigma_{\max }^{2}\right)} \cdot \frac{\xi}{4 L}, \forall x \notin[-\tau, \tau],
$$

in which case $\int_{[-\tau, \tau]^{c}} x^{p} \cdot \mathcal{F}(x) \mathrm{d} x$ can be written as

$$
\begin{aligned}
\sum_{i=1}^{k} p_{i} & \int_{[-\tau, \tau]^{c}} x^{p} \cdot \frac{1}{\sqrt{2 \pi} \sigma_{i}} \cdot e^{-x^{2} /\left(2 \sigma_{i}^{2}\right)} \mathrm{d} x \\
& \leq L \cdot \int_{[-\tau, \tau]^{c}} \frac{1}{\sqrt{2 \pi} \sigma_{\max }} \cdot e^{-x^{2} /\left(4 \sigma_{\max }^{2}\right)} \cdot \frac{\xi}{4 L} \mathrm{~d} x \leq \xi / 2 .
\end{aligned}
$$

To reach (3), we want $p \ln x \leq \frac{x^{2}}{4 \sigma_{\max }^{2}}-\ln (4 L / \xi), \forall x \notin[-\tau, \tau]$. For $x \geq 8 \sigma_{\max }^{2} \ln (4 L / \xi)$, we get that $\frac{x^{2}}{4 \sigma_{\max }^{2}}-\ln (4 L / \xi) \geq \frac{x^{2}}{8 \sigma_{\max }^{2}}$, and for $x \geq 8 p \sigma_{\max }^{2}$, we have that $p \ln x \leq \frac{x^{2}}{8 \sigma_{\max }^{2}}$. We conclude that for $\tau=8 \bar{\sigma}^{2} \cdot \max \{p, \ln (4 L / \xi)\}$, (3) holds. We get

$$
\begin{gathered}
\left|\int_{[-\tau, \tau]^{c}} x^{p} \cdot \mathcal{F}(x) \mathrm{d} x\right|+\left|\int_{-\tau}^{\tau} x^{p} \cdot(\mathcal{F}-\mathcal{G})(x) \mathrm{d} x\right| \leq \xi / 2+ \\
\left(\int_{-\tau}^{\tau} x^{2 p} \mathrm{~d} x\right)^{1 / 2} \cdot\|\mathcal{F}-\mathcal{G}\|_{2} \leq \xi / 2+\left(\frac{2 \tau^{2 p+1}}{2 p+1} \eta\right)^{1 / 2} \leq \eta .
\end{gathered}
$$

completing the proof.

We now show how to estimate the minimum variance by taking high moments of the Fourier transform of our $L_{2}$-close estimator. 

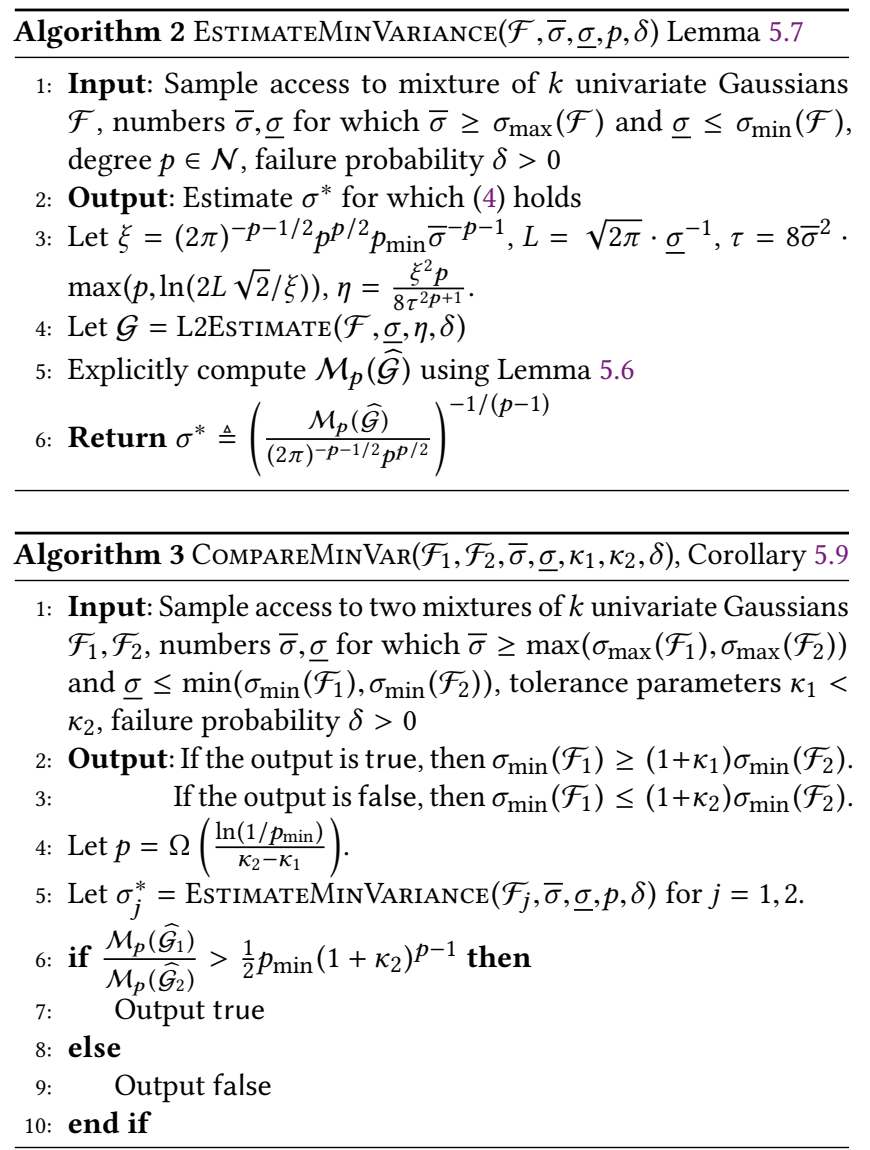

As an important subroutine, we show that it is efficient to compute the Fourier moments of our density estimate, by using the fact that it is piecewise polynomial. Specifically:

Lemma 5.6. Given a s-piece degree-d polynomial $p: \mathbb{R} \rightarrow \mathbb{R}$, and any $\tau>0$ and nonnegative integer $\ell>0$, there is an algorithm which runs in time $O\left(s \ell d^{3}\right)$ and which outputs $\int_{-\tau}^{\tau} \widehat{p}[\omega] \omega^{\ell} \mathrm{d} \omega$.

We defer the description of this algorithm as well as the proof of correctness to the full version. We now show:

LEMma 5.7. Let $\mathcal{F}$ be a mixture of $k$ univariate zero-mean Gaussians with parameters $\left(\left\{p_{i}\right\}_{i \in[k]},\left\{\sigma_{i}\right\}_{i \in[k]}\right)$. Let $\bar{\sigma} \geq \sigma_{\max }(\mathcal{F}), \sigma \leq$ $\sigma_{\min }(\mathcal{F})$. EstimateMinVARIANCE $(p, \mathcal{F}, \bar{\sigma}, \underline{\sigma}, \delta)($ Algorithm 2) takes $N=p_{\min }^{-4} k \ln (1 / \delta) \cdot \operatorname{poly}\left(\bar{\sigma}, p, \ln \left(1 / p_{\min }\right), \ln (1 / \underline{\sigma})\right)^{O(p)}$ samples, runs in time $\widetilde{O}(N)$, and outputs a number $\sigma^{*}$ for which

$$
\left(\frac{3}{4}\right)^{1 /(p-1)} \cdot \sigma_{\min }(\mathcal{F}) \leq \sigma^{*} \leq\left(\frac{3}{2 p_{\min }}\right)^{1 /(p-1)} \cdot \sigma_{\min }(\mathcal{F})
$$

with probability at least $1-\delta$.

Proof. The runtime and sample bounds for general $p$ just follow from the fact that these quantities are dominated by the cost of running L2Estimate $(\mathcal{F}, \underline{\sigma}, \eta, \delta)$ for our choice of $\eta$ in EstimateMInVARIANCE. By Corollary 5.4, if we run L2Estimate $(\mathcal{F}, \underline{\sigma}, \eta, \delta)$ and produce $\mathcal{G}$, we know that $\|\mathcal{F}-\mathcal{G}\|_{2}^{2} \leq \eta$. By Plancherel's, $\|\widehat{\mathcal{F}}-\widehat{\mathcal{G}}\|_{2}^{2} \leq \eta$. To apply Lemma 5.5 , note that $\widehat{\mathcal{F}}$ is an affine linear combination of Gaussians $\sum_{i=1}^{k} p_{i} \frac{1}{\sqrt{2 \pi} \sigma_{i}} \mathcal{N}\left(0, \frac{1}{4 \pi^{2} \sigma_{i}^{2}}, \omega\right)$, and its coefficients sum to $\sum_{i=1}^{k} p_{i} \cdot \frac{1}{\sqrt{2 \pi} \sigma_{i}} \leq \frac{1}{\sqrt{2 \pi} \sigma_{\min }(\mathcal{F})} \leq L$ by our choice of $L$ in EstimateMinVARIANCE.

So by Lemma 5.5, if we define $\widehat{\mathcal{G}}^{\prime}$ by $\widehat{\mathcal{G}}^{\prime}(x)=\mathbb{1}[x \in[-\tau, \tau]]$. $\widehat{\mathcal{G}}(x)$, then we get that $\left|\mathcal{M}_{p}(\widehat{\mathcal{F}})-\mathcal{M}_{p}\left(\widehat{\mathcal{G}}^{\prime}\right)\right| \leq \xi$. Furthermore, note that $\mathcal{M}_{p}(\widehat{F})$ is at most $\sum_{i=1}^{k} p_{i} \cdot \frac{1}{\sqrt{2 \pi} \sigma_{i}} \cdot p^{p / 2} \cdot\left(\frac{1}{4 \pi^{2}\left(\sigma_{i}\right)^{2}}\right)^{p / 2}=$ $(2 \pi)^{-p-1 / 2} p^{p / 2} \sum_{i=1}^{k} p_{i}\left(\sigma_{i}\right)^{-p-1}$. If we had $\xi=(2 \pi)^{-p-1 / 2} p^{p / 2} \xi^{\prime}$ for some $\xi^{\prime}>0$, then we get that

$$
\mathcal{M}_{p}(\widehat{\mathcal{G}})=(2 \pi)^{-p-1 / 2} p^{p / 2}\left[\sum_{i=1}^{k} p_{i}\left(\sigma_{i}\right)^{-p-1} \pm \xi^{\prime}\right] .
$$

For $\xi^{\prime} \triangleq \frac{1}{3} p_{\min } \bar{\sigma}^{-p-1},\left(\sum_{i=1}^{k} p_{i}\left(\sigma_{i}\right)^{-p-1}\right)^{-1 /(p-1)}$ lies in $\sigma_{\min }(\mathcal{F})$. $\left[1, p_{\min }^{-1 /(p-1)}\right]$, so $\sigma^{*} \triangleq\left(\frac{\mathcal{M}_{p}(\widehat{\mathcal{G}})}{(2 \pi)^{-p-1 / 2} p^{p / 2}}\right)^{-1 /(p-1)}$ satisfies (4).

For the last part of the lemma, take $p=20 \ln \left(\frac{3}{2 p_{\min }}\right)+1 \geq 4$.

We now identify two specific parameter settings for this algorithm which will be useful later on. First, if we take the degree $p$ to be relatively small, we are able to get a constant approximation to the minimum variance very efficiently:

Corollary 5.8. Let $p=\Theta\left(\ln \left(1 / p_{\min }\right)\right)$. Then, the algorithm ESTIMATEMINVARIANCE $(p, \mathcal{F}, \bar{\sigma}, \underline{\sigma}, \delta)$ has sample and time complexity $\widetilde{O}\left(p_{\min }^{-4} k \ln (1 / \delta) \cdot \operatorname{poly}\left(\bar{\sigma}, p, \ln \left(1 / p_{\min }\right), \ln (1 / \underline{\sigma})\right)^{O(p)}\right)$, and the output $\sigma^{*}$ is a 1.1-approximation of $\sigma_{\min }(\mathcal{F})$.

We also have:

Corollary 5.9. Let $0<\kappa_{1}<\kappa_{2} \leq 1$, and let $\mathcal{F}_{1}$ and $\mathcal{F}_{2}$ be two univariate zero-mean $k$-GMMs with parameters $\left(\left\{p_{i}\right\},\left\{\sigma_{i}^{(1)}\right\}\right)$ and $\left(\left\{p_{i}\right\},\left\{\sigma_{i}^{(2)}\right\}\right)$ respectively. Let $\bar{\sigma} \geq \max \left(\sigma_{\max }\left(\mathcal{F}_{1}\right), \sigma_{\max }\left(\mathcal{F}_{2}\right)\right), \underline{\sigma} \leq$ $\min \left(\sigma_{\min }\left(\mathcal{F}_{1}\right), \sigma_{\min }\left(\mathcal{F}_{2}\right)\right)$. Then, with probability $1-\delta$, the algorithm COMPAREMINVAR $\left(\mathcal{F}_{1}, \mathcal{F}_{2}, \bar{\sigma}, \underline{\sigma}, \kappa_{1}, \kappa_{2}, \delta\right)$ (Algorithm 3) satisfies:

- If $\sigma_{\min }\left(\mathcal{F}_{1}\right) \geq\left(1+\kappa_{2}\right) \sigma_{\min }\left(\mathcal{F}_{2}\right)$, then it outputs true.

- If $\sigma_{\min }\left(\mathcal{F}_{1}\right) \leq\left(1+\kappa_{1}\right) \sigma_{\min }\left(\mathcal{F}_{2}\right)$, then it outputs false.

This takes $N=p_{\min }^{-4} k \ln (1 / \delta) \cdot \operatorname{poly}\left(\bar{\sigma},\left(\kappa_{2}-\kappa_{1}\right)^{-1}, \ln \left(1 / p_{\min }\right)\right.$, $\ln (1 / \underline{\sigma}))^{O\left(\left(\kappa_{2}-\kappa_{1}\right)^{-1} \ln \left(1 / p_{\min }\right)\right)}$ samples and runs in time $\widetilde{O}(N)$.

\subsection{Moment Descent}

In this section we will show how to obtain a warm start using the COMPAREMINVAR subroutine of the previous section.

The first ingredient we need is a subroutine to estimate $\operatorname{span}\left(\left\{w_{i}-\right.\right.$ $\left.a\}_{i \in[k]}\right)$, where $a$ is our current guess for a direction. For any $x, y, a \in \mathbb{R}^{d}$, define the matrix

$$
\mathbf{M}_{a}^{x, y} \triangleq \frac{1}{2}\left[(y-\langle a, x\rangle)^{2} x x^{\top}-(y-\langle a, x\rangle)^{2} \cdot \mathbf{I}\right] \in \mathbb{R}^{d \times d}
$$

and let

$$
\widehat{\mathbf{M}}_{a}^{(N)} \triangleq \frac{1}{N} \sum_{i=1}^{N} \mathbf{M}_{a}^{x_{i}, y_{i}}
$$

for $\left(x_{1}, y_{1}\right), \ldots,\left(x_{N}, y_{N}\right)$ i.i.d. samples from $\mathcal{D}$. Notice there is a matrix-vector oracle for $\widehat{\mathbf{M}}_{a}^{(N)}$ which runs in time $O(N d)$. 
LEMMA 5.10. Let $\mathcal{D}$ be a mixture of $k$ spherical linear regressions. For any $a \in \mathbb{R}^{d}, \mathbb{E}_{(x, y) \sim \mathcal{D}}\left[\mathbf{M}_{a}^{x, y}\right]=\sum_{i=1}^{k} p_{i}\left(w_{i}-a\right)\left(w_{i}-a\right)^{\top}$. For $\beta, \delta>0$ and $\left.N=\widetilde{\Omega}\left(\max _{i \in[k]}\left\|w_{i}-a\right\|_{2}^{2} \cdot p_{\min }^{-1} \cdot \beta^{-2} \cdot d \cdot \ln (k / \delta)\right)\right)$, we have that $\operatorname{Pr}\left[\left\|\widehat{\mathbf{M}}_{a}^{(N)}-\mathbb{E}_{(x, y) \sim \mathcal{D}}\left[\mathbf{M}_{a}^{x, y}\right]\right\|_{2} \geq \beta\right] \leq \delta$.

We emphasize that $\mathbb{E}\left[\mathbf{M}_{a}^{x, y}\right]$ is the same regardless of $\eta$, but the value of $\eta$ will slightly affect concentration, though not in the regimes in which we will apply Lemma 5.10. We defer the proof of this to the full version. Combining this with power method allows us to quantify the effectiveness of approximate $k$-SVD of an empirical estimate for $\mathbb{E}\left[\mathbf{M}_{a}^{x, y}\right]$ for capturing the span of the $w_{i}$ :

LemmA 5.11. Let $N=\widetilde{\Omega}\left(\frac{\max _{i \in[k]}\left\|w_{i}-a\right\|_{2}^{2}}{\min _{i \in[k]}\left\|w_{i}-a\right\|_{2}^{2}} \cdot p_{\min }^{-2} \cdot k^{2} d \ln (k / \delta)\right)$. There is an algorithm APPROXBLOCKSVD that runs in time $\widetilde{O}(k \cdot N \cdot d)$ and outputs $\mathbf{U}$ so that with probability $1-\delta, 1 / 2 \leq \frac{\left\|\mathrm{U}^{\top}\left(w_{i}-a\right)\right\|_{2}}{\left\|w_{i}-a\right\|_{2}} \leq 1$.

We are now ready to analyze the amount of progress each step of moment descent makes.

Lemma 5.12. For any $\delta>\exp (-\sqrt{k})$, the following holds. Let $\sigma_{t}^{2} \triangleq \min _{i \in[k]}\left\|w_{i}-a_{t}\right\|_{2}$. Denote the minimizing index $i$ by $i^{*}$. For $M \triangleq e^{\sqrt{k}} \ln (2 / \delta)$ and $g_{1}, \ldots, g_{M} \sim \mathcal{N}\left(0, \mathbf{I}_{k}\right)$, let $v_{j}=\frac{\mathrm{U} g_{i}}{\left\|\mathrm{U} g_{i}\right\|_{2}} \in \mathbb{S}^{d-1}$ for $j \in[M]$. Let $\sigma^{*}$ satisfy $0.9 \sigma_{t} \leq \sigma^{*} \leq 1.1 \sigma_{t}$. Let $\eta=\frac{1}{2} k^{-1 / 4} \sigma^{*}$. With probability at least $1-\delta$,1) there exists $j \in[M]$ for which $\left\|w_{i^{*}}-a_{t}-\eta \cdot v_{j}\right\|_{2}^{2} \leq\left(1-\frac{1}{5 \sqrt{k}}\right) \sigma_{t}^{2}$, and 2) for all $j \in[M]$ and $i \in[k],\left\|w_{i}-a_{t}-\eta \cdot v_{j}\right\|_{2}^{2} \geq\left(1-\frac{9}{\sqrt{k}}\right) \sigma_{t}^{2}$.

Proof. For any $i \in[k]$, we may write

$$
\left\|w_{i}-a_{t}-\eta v_{j}\right\|_{2}^{2}=\left\|w_{i}-a_{t}\right\|_{2}^{2}+\eta^{2}-2 \eta\left\langle w_{i}-a_{t}, v_{j}\right\rangle .
$$

Define $\widetilde{w}_{i} \triangleq \frac{w_{i}-a_{t}}{\left\|w_{i}-a_{t}\right\|_{2}}$. For every $j \in[M]$, let $A_{j}$ be the event that $\left\langle v_{j}, \widetilde{w}_{i^{*}}\right\rangle \geq \frac{1}{2} k^{-1 / 4}$. For every $j \in[M]$ and $i \in[k]$, let $B_{j}[i]$ be the event that $\left\langle v_{j}, \widetilde{w}_{i}\right\rangle \leq 3 k^{-1 / 4}$. We would like to condition on the event that $\mathcal{E} \triangleq\left(\vee_{j \in[M]} A_{j}\right) \wedge\left(\bigwedge_{i \in[k], j \in[M]} B_{j}[i]\right)$.

We first verify that conditioned on $\mathcal{E}, 1)$ and 2 ) hold. There is at least one $j \in[M]$ for which

$$
\begin{aligned}
& \left\|w_{i^{*}}-a_{t}-\eta v_{j}\right\|_{2}^{2} \leq\left\|w_{i^{*}}-a_{t}\right\|_{2}^{2}+\eta^{2}-\eta \sigma_{t} \cdot k^{-1 / 4} \leq \\
& \left(1+\frac{1}{4}\left(\left(\frac{\sigma^{*}}{\sigma_{t}}\right)^{2}-2\left(\frac{\sigma^{*}}{\sigma_{t}}\right)\right) k^{-1 / 2}\right) \sigma_{t}^{2} \leq\left(1-\frac{1}{5 \sqrt{k}}\right) \sigma_{t}^{2} .
\end{aligned}
$$

where the first step follows from $A_{j}$, and the third follows from $\sigma^{*} / \sigma_{t} \in[0.9,1.1]$. For every $i \in[k], j \in[M]$ we have that

$\left\|w_{i}-a_{t}-\eta v_{j}\right\|_{2}^{2} \geq\left\|w_{i}-a_{t}\right\|^{2}+\eta^{2}-6 \eta \sigma_{t} \cdot k^{-1 / 4} \geq\left(1-\frac{9}{\sqrt{k}}\right) \sigma_{t}^{2}$,

where the first step follows from $B_{j}[i]$.

We defer the proof that $\operatorname{Pr}[\mathcal{E}] \geq 1-\delta$, which follows by standard concentration for random unit vectors, to the full version.

By carefully assembling the lemmas from this and the previous section, we obtain the following, which implies Theorem 5.1 upon closely tracking the runtime and sample complexity. We refer to the full version for details, including how to use FourierMomentDescent to "peel" off components and learn all $k$ regressors.
Lemma 5.13. There is an absolute constant $C>0$ for which the following holds. Let $\mathcal{D}$ be a mixture of spherical linear regressions with mixing weights $\left\{p_{i}\right\}$, directions $\left\{w_{i}\right\}$, and noise rate $\varsigma$. For any $\epsilon, \delta>0$ and $\varsigma^{2} \leq \epsilon^{2} / 10$, with probability at least $1-\delta$, FOURIERMOMENTDESCENT(D) $\delta, \epsilon)$ (Algorithm 4) outputs direction $a_{T} \in \mathbb{R}^{d}$ for which $\min _{i \in[k]}\left\|w_{i}-a_{T}\right\|_{2} \leq \epsilon$.

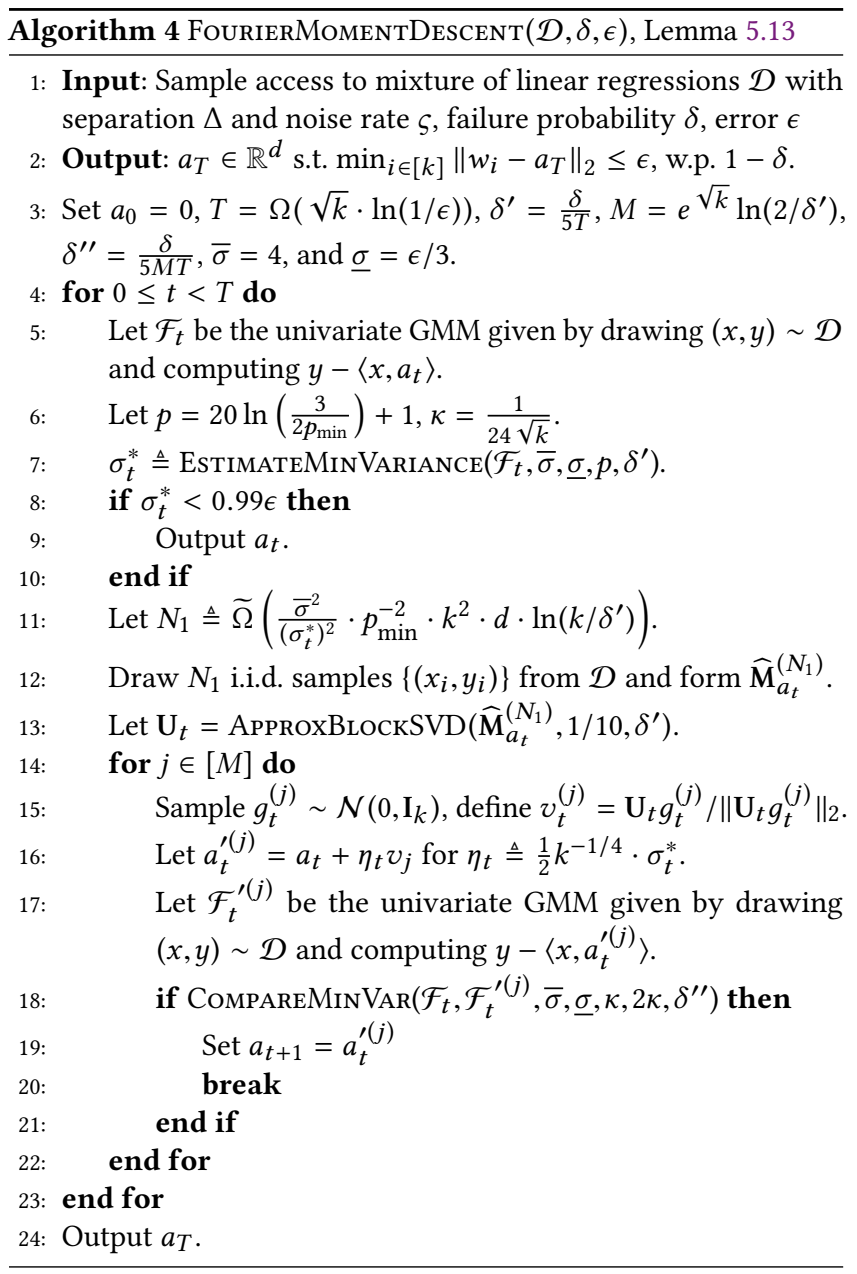

\section{LEARNING ALL COMPONENTS UNDER NOISE}

We now describe how to learn all components under the much more challenging setting with regression noise. We show that, at the extra cost of running in time exponential in $1 / \Delta^{2}$ in addition to $\sqrt{k}$, there is an algorithm, which we call LEARNWITHNoISE, that can learn mixtures of linear regressions to error $\epsilon$ when $\varsigma=O(\epsilon)$.

Theorem 6.1. Given $\delta, \epsilon>0$ and a mixture of spherical linear regressions $\mathcal{D}$ with regressors $\left\{w_{1}, \ldots, w_{k}\right\}$, separation $\Delta$, and noise $\varsigma=O(\epsilon)$, with probability $1-\delta$, LEARNWITHNOISE $(\mathcal{D}, \delta, \epsilon)$ returns a list of vectors $\mathcal{L} \triangleq\left\{\widetilde{w}_{1}, \ldots, \widetilde{w}_{k}\right\}$ for which there is a permutation $\pi$ : $[k] \rightarrow[k]$ for which $\left\|\tilde{w}_{i}-w_{\pi(i)}\right\|_{2} \leq \epsilon$ for all $i \in[k]$. Furthermore, 
LEARNWITHNOISE requires $N=\widetilde{O}\left(d \epsilon^{-2} \ln (1 / \epsilon) \ln (1 / \delta) p_{\min }^{-4} \Delta^{-2}\right.$. $\left.\operatorname{poly}\left(k, 1 / \epsilon, \ln \left(1 / p_{\min }\right)\right)^{O\left(\sqrt{k} \ln \left(1 / p_{\min }\right) / \Delta^{2}\right)}\right)$ samples and time $N d$. poly $\log \left(k, d, 1 / \Delta, 1 / p_{\min }, 1 / \epsilon\right)$.

In Section 6.1 we prove the key technical ingredient behind our proof of Theorem 6.1, Lemma 6.4, which allows us to carefully control the dynamics of Fourier moment descent. In Section 6.2 we describe how to get an initialization which satisfies the hypotheses of Lemma 6.4. In Section 6.3 we sketch our algorithm LEARNWITHNoIse, deferring the details to the full version.

\subsection{Staying on the Same Component}

The main result of this section and the primary technical component behind Theorem 6.1 is Lemma 6.4 below. This is a substantially more refined version of Lemma 5.12 in which we control not only the probability we make progress in the $t$-th step of moment descent, but also the probability that the the component $a_{t+1}$ is closest to is the same as the one $a_{t}$ is closest to.

We first introduce some preliminary notation and facts that we will use in the proof of Lemma 6.4.

For $v \in \mathbb{S}^{d-1}$, define $\mathcal{F}$ and $\mathcal{F}_{v}^{\prime}$ respectively to be the distribution of $y-\left\langle a_{t}, x\right\rangle$ and of $y-\left\langle a_{t}+\eta v, x\right\rangle$, where $(x, y) \sim \mathcal{D}$. Let $\sigma_{t}^{2} \triangleq$ $\varsigma^{2}+\min _{i \in[k]}\left\|w_{i}-a_{t}\right\|_{2}$. Denote the minimizing index $i$ by $i^{*}$.

We record the following application of Lemma 5.10 and power method which says we have access to $\operatorname{span}\left(\left\{w_{i}-a_{t}\right\}\right)$ up to $1 / \operatorname{poly}(k)$ additive error.

Lemma 6.2. Let $\delta_{\text {samp }}, \delta^{\prime}>0$ and $a_{t} \in \mathbb{R}^{d}$. If we draw $N_{1}=$ $\widetilde{\Omega}\left(p_{\text {min }}^{-1} \delta_{\text {samp }}^{-1} \cdot d \cdot \ln \left(k / \delta^{\prime}\right)\right)$ samples, form $\widehat{\mathbf{M}}_{a_{t}}^{\left(N_{1}\right)} \in \mathbb{R}^{d \times d}$ as defined in (5), and run APPROXBLOCKSVD $\left(\widehat{\mathbf{M}}_{a_{t}}^{\left(N_{1}\right)}, \delta_{\text {samp }}, \delta^{\prime}\right)$ to produce a matrix $\mathrm{U} \in \mathbb{R}^{k \times d}$, then with probability $1-\delta^{\prime}$ we have that for any $a, b \in \mathbb{S}^{d-1}$ in the row span of $\mathrm{U}$,

(1) $\langle\mathbf{U} a, \mathbf{U} b\rangle \leq\langle a, b\rangle-\delta_{\text {samp }}$

(2) $1-\delta_{\text {samp }} \leq\left\|\mathrm{U}\left(w_{i}-a_{t}\right)\right\|_{2} \leq 1-\delta_{\text {samp. }}$.

Lastly, the following elementary fact will be useful:

FACT 6.3. If $x \in \mathbb{R}_{\geq 0}$ satisfies $\frac{1}{2}\left(x+x^{-1}\right) \geq 1+\beta^{2}$ for some $0<\beta \leq 1$, then $1-\beta / 2 \leq x \leq 1+\beta / 2$.

We are now in a position to state and prove our main result of this section, Lemma 6.4. This lemma roughly says that if we sample $M=\exp \left(\Omega\left(\sqrt{k} / \Delta^{2}\right)\right)$ random steps $v_{1}, \ldots, v_{M}$ at time $t$ of moment descent, then with high probability, if $j^{*} \in[M]$ is the first index on which COMPAREMinVAR outputs true, then not only does walking in direction $v_{j^{*}}$ contract $\sigma_{t}$ by a factor of $1-\Omega(\Delta / \sqrt{k})$ with high probability, but additionally, with at least $1 / \operatorname{poly}(k)$ probability, it also keeps us closest to the component we were already closest to, in the following robust sense. Specifically, if we have a $\left(1+\Omega\left(\Delta^{2} \sqrt{k}\right)\right)$ gap between $\left\|w_{i^{*}}-a_{t}\right\|_{2}$ and all other $\left\|w_{i}-a_{t}\right\|_{2}$, then with at least $1 / \operatorname{poly}(k)$ probability, after one more iteration of moment descent, the $i^{*}$-th component will still be the closest to our new guess $a_{t+1} \in \mathbb{R}^{d}$, and this gap will persist.

LEMMA 6.4. There exist constants $a_{\mathrm{LR}}, a_{\text {trials }}, a_{\text {scale }}$, constants $\bar{\beta}>$ $\beta$, a constant $0 \leq a_{\text {noise }} \leq 1 / 5$, and a constant $\tau_{\text {gap }}>0$, such that for all $c<\tau_{\text {gap }}$, the following holds for some $0<\kappa_{1}<\kappa_{2} \leq 1$ satisfying $\kappa_{2}-\kappa_{1}=c \Delta^{2} k^{-1 / 2}$.
Let $\delta>0$. Suppose that $\varsigma^{2} \leq a_{\text {noise }} \cdot \epsilon^{2}$. Suppose that

$$
\left\|w_{i}-a_{t}\right\|_{2} \leq a_{\text {scale }} \cdot k^{1 / 4}
$$

for all $i \in[k]$. For $M \triangleq e^{a_{\text {trials }} \sqrt{k} / \Delta^{2}} \ln (3 / \delta)$ and $g_{1}, \ldots, g_{M} \sim \mathcal{N}(0, \mathbf{I})$, let $v_{j}=\frac{g_{j} \mathrm{U}}{\left\|g_{j} \mathrm{U}\right\|_{2}} \in \mathbb{S}^{d-1}$ for $j \in[M]$. Let $\sigma^{*}$ be a number for which $0.9 \sigma_{t} \leq \sigma^{*} \leq 1.1 \sigma_{t}$, and let $\eta \triangleq a_{\mathrm{LR}} \cdot \Delta \cdot \sigma_{*} \cdot k^{-1 / 4}$.

Then with probability at least $1-\delta$ over the randomness of $g_{1}, \ldots, g_{M}$ as well as over the behavior of all runs of COMPAREMINVAR, the following events hold:

(1) (Progress detected) If $\min _{i \in[k]}\left\|w_{i}-a_{t}\right\|_{2}^{2} \geq \epsilon^{2} / 2$, then $\operatorname{COMPAREMINVAR}\left(\mathcal{F}, \mathcal{F}_{v}^{\prime}, a_{\text {scale }} \cdot k^{-1 / 4}, \sigma^{*} / 1.1, \kappa_{1}, \kappa_{2}, \delta / 3 M\right)$ outputs true for at least one $j \in[M]$.

Let $j^{*}$ be the smallest such $j$, and define $a_{t+1} \triangleq a_{t}+\eta v_{j^{*}}$.

(2) (Make some progress) If $\min _{i \in[k]}\left\|w_{i}-a_{t}\right\|_{2}^{2} \geq \epsilon^{2} / 2$, then we have $\sigma_{t+1}^{2} \leq\left(1-\beta \Delta^{2} / \sqrt{k}\right) \sigma_{t}^{2}$.

(3) (But not too much) Regardless of whether $\min _{i}\left\|w_{i}-a_{t}\right\|_{2}^{2} \leq$ $\epsilon^{2} / 2$, we have $\sigma_{t+1}^{2} \geq\left(1-\bar{\beta} \Delta^{2} / \sqrt{k}\right) \sigma_{t}^{2}$.

If we assume that for all $i \neq i^{*}$,

$$
\left\|w_{i}-a_{t}\right\|_{2} \geq\left(1+c \Delta^{2} / \sqrt{k}\right) \cdot\left\|w_{i^{*}}-a_{t}\right\|_{2},
$$

then crucially, we have that with probability $1 / \operatorname{poly}(k)$, the events above hold and additionally:

(4) $\left(i^{*}\right.$ remains closest $\mathbf{w} /$ same gap) If $\min _{i}\left\|w_{i}-a_{t}\right\|_{2}^{2} \geq$ $\epsilon^{2} / 2$, then for all $i \neq i^{*},\left\|w_{i}-a_{t}-\eta v_{j^{*}}\right\|_{2} \geq\left(1+c \Delta^{2} / \sqrt{k}\right)$. $\left\|w_{i^{*}}-a_{t}-\eta v_{j^{*}}\right\|_{2}$.

We emphasize the main content is (4). In the sequel, we state some claims without proof, deferring to the full version.

Proof. Henceforth we will say that "CompareMinVAr succeeds and outputs true/false on direction $v$ " to mean that a single run of

$\operatorname{CompareMinVAR}\left(\mathcal{F}, \mathcal{F}_{v}^{\prime}, a_{\text {scale }} \cdot k^{-1 / 4}, \sigma^{*} / 1.1, \kappa_{1}, \kappa_{2}, \delta / 3 M\right)$

is successful (in the language of Corollary 5.9, this happens with probability $1-\delta / 3 M)$ and outputs true/false.

Recall (6). Define $\delta_{i} \triangleq w_{i}-a_{t}$ and $\widehat{\delta}_{i} \triangleq \frac{w_{i}-a_{t}}{\left\|w_{i}-a_{t}\right\|_{2}}$. For every $i \neq i^{*}$, define $\delta_{i}^{\perp} \triangleq \widehat{\delta}_{i}-\left\langle\widehat{\delta}_{i^{*}}, \widehat{\delta}_{i}\right\rangle \widehat{\delta}_{i^{*}}$. Finally, let $\gamma_{i}^{(j)}=\left\langle\widehat{\delta}_{i}, v_{j}\right\rangle$. Where the context is clear, we will omit the superscript $(j)$.

Let $v_{A}, v_{B}, v_{C}>0$ be absolute constants, and suppose $v_{A}<v_{B}$. For $i \in[k]$ and $j \in[M]$, define the following events:

(1) Let $A_{j}[i]$ be the event that $\gamma_{i^{*}}^{(j)} \geq v_{A} \Delta k^{-1 / 4}$.

(2) Let $B_{j}[i]$ be the event that $\gamma_{i}^{(j)} \leq v_{B} \Delta k^{-1 / 4}$.

(3) Let $C_{j}$ be the event that $A_{j}\left[i^{*}\right]$ occurs and also $\left\langle v_{j}, \delta_{i}^{\perp}\right\rangle \leq$ $v_{C} \Delta^{2} k^{-1 / 2}\left\|\delta_{i}^{\perp}\right\|_{2}$ for all $i \neq i^{*}$.

For $j \in[M]$, let $B_{j}$ denote the event that $B_{j}[i]$ occurs for all $i$.

By our assumption on $\sigma_{*}$ and the definition of $\eta$, we know that $\eta=a_{\mathrm{LR}}^{\prime} \cdot k^{-1 / 4} \cdot\left\|\delta_{i}\right\|_{2}$, where $a_{\mathrm{LR}}^{\prime} \in[0.9,1.1] \cdot a_{\mathrm{LR}}$. It will be useful later in the proof to assume that $v_{B}<a_{\mathrm{LR}}^{\prime}<2 v_{A}$.

First, we can compute the exact distance to $v_{i^{*}}$ after walking along $v_{j}$ and, given $B_{j}[i]$, lower bound the distances to all other $v_{i}$.

Claim 6.5. Let $i \in[k], j \in[M]$, and suppose $B_{j}[i]$ occurs. Then

$$
\left\|\delta_{i}-\eta v_{j}\right\|_{2}^{2} \geq\left\|\delta_{i}\right\|_{2}^{2} \cdot\left(1+a_{\mathrm{LR}}^{2} k^{-1 / 2}-2 a_{\mathrm{LR}}^{\prime} k^{-1 / 4} \gamma_{i}^{(j)}\right),
$$


with equality when $i=i^{*}$. Furthermore, (7) implies that

$$
\begin{array}{r}
\left\|\delta_{i}-\eta v_{j}\right\|_{2}^{2} \geq\left\|\delta_{i^{*}}\right\|_{2}^{2} \cdot\left(\left(1+c \Delta^{2} k^{-1 / 2}\right)^{2}+a_{\mathrm{LR}}^{\prime 2} \Delta^{2} k^{-1 / 2}\right. \\
\left.-2 a_{\mathrm{LR}}^{\prime} \Delta k^{-1 / 4} \gamma_{i}^{(j)}-2 c a_{\mathrm{LR}}^{\prime} \Delta^{3} k^{-3 / 4} \gamma_{i}^{(j)}\right) .
\end{array}
$$

when $i \neq i^{*}$.

Using (8) of Claim 6.5, which is an equality when $i=i^{*}$, we can upper bound the distance to $v_{i^{*}}$ after walking along $v_{j}$, provided events $A_{j}\left[i^{*}\right]$ and $B_{j}[i]$ occur.

CLAIM 6.6. Let $j \in[M]$, and suppose $A_{j}\left[i^{*}\right]$ and $B_{j}\left[i^{*}\right]$ occur. There is an absolute $\underline{\beta}^{\prime}>0$ s.t. $\left\|\delta_{i^{*}}-\eta v_{j}\right\|_{2}^{2} \leq\left\|\delta_{i^{*}}\right\|_{2}^{2} \cdot\left(1-\underline{\beta}^{\prime} \Delta^{2} k^{-1 / 2}\right)$.

Next, using (9) of Claim 6.5, we argue that the only way to make progress towards a different component $i \neq i^{*}$ by an amount comparable to that of Claim 6.6, is if $A_{j}[i]$ has occurred. In particular, the following claim is the contrapositive of this.

Claim 6.7. Let $i \neq i^{*}$ and $j \in[M]$, and suppose $B_{j}[i]$ occurs and $A_{j}[i]$ does not. Then $\left\|\delta_{i}-\eta v_{j}\right\|_{2}^{2} \geq\left\|\delta_{i^{*}}\right\|_{2}^{2} \cdot\left(1-\left(\underline{\beta}^{\prime}-c\right) \Delta^{2} k^{-1 / 2}\right)$.

Henceforth, let $\kappa_{1}=\left(\underline{\beta}^{\prime}-\frac{3 c}{2}\right) \Delta^{2} k^{-1 / 2}, \kappa_{2}=\left(\underline{\beta}^{\prime}-\frac{c}{2}\right) \Delta^{2} k^{-1 / 2}$. In Lemma 6.4, we will take $\underline{\beta} \triangleq \underline{\beta}^{\prime}-\frac{3 c}{2}$.

Claims 6.6 and 6.7 now imply the following about the behavior of COMPAREMINVAR. The upshot of the following two corollaries is that for any $j \in[M]$, if $B_{j}[i]$ occurs for every $i$ and COMPAREMINVAR succeeds and outputs true on direction $v_{j}$, the conditional probability of $A_{j}\left[i^{*}\right]$ happening is at least the conditional probability of $A_{j}[i]$ happening for any $i \neq i^{*}$.

Corollary 6.8. Let $j \in[M]$, and suppose $A_{j}\left[i^{*}\right]$ and $B_{j}\left[i^{*}\right]$ occur. Then COMPAREMINVAR succeeds and outputs true on direction $v_{j}$.

Corollary 6.9. Let $i \neq i^{*}$ and $j \in[M]$, and suppose $B_{j}[i]$ holds and COMPAREMINVAR succeeds and outputs true on direction $v_{j}$. Then $A_{j}[i]$ has also occurred.

We also give an upper bound to the amount of progress that any $v_{j}$ could make in any direction $i$, provided $B_{j}[i]$ holds.

Claim 6.10. Let $i \in[k], j \in[M]$, and suppose $B_{j}[i]$ occurs. There is an absolute $\bar{\beta}>\underline{\beta}$ s.t. $\left\|\delta_{i}-\eta v_{j}\right\|_{2}^{2} \geq\left\|\delta_{i^{*}}\right\|_{2}^{2} \cdot\left(1-\bar{\beta} \Delta^{2} k^{-1 / 2}\right)$.

At this point, we could already use Corollary 6.8 and Claim 6.10, together with straightforward bounds on the probabilities of $A_{j}\left[i^{*}\right]$ and $B_{j}[i]$ (see Claims 6.12 and 6.13 below) to show parts 1), 2), and 3 ) of the lemma. Note that the proofs of these do not use (7), so in particular they hold with the claimed probability without (7).

We next lay the foundation for showing part 4) of the lemma holds with at least $1 / \operatorname{poly}(k)$ probability, assuming (7). Thus far we have not talked about the events $C_{j}$. It is at this point that we arrive at the main claim of the proof, namely that if event $C_{j}$ happens, then the gap of (7) between the $i^{*}$-th component and all other components persists in the next step.

Claim 6.11. Let $j \in[M]$ and suppose $C_{j}$ and $B_{j}[i]$ occur for all $i \in[k]$. Then $\left\|\delta_{i}-\eta v_{j}\right\|_{2} \geq\left(1+c \Delta^{2} k^{-1 / 2}\right) \cdot\left\|\delta_{i^{*}}-\eta v_{j}\right\|_{2}$ for all $i \neq i^{*}$.
Proof. Suppose we could show that

$$
\gamma_{i}^{(j)} \leq \gamma_{i^{*}}^{(j)}
$$

for all $i \neq i^{*}$. Then by (8), $\frac{\left\|\delta_{i}-\eta v_{j}\right\|_{2}^{2}}{\left\|\delta_{i^{*}}-\eta v_{j}\right\|_{2}^{2}}$ would be at least

$$
\frac{\left\|\delta_{i}\right\|^{2}}{\left\|\delta_{i^{*}}\right\|_{2}^{2}} \cdot \frac{1+a_{\mathrm{LR}}^{\prime 2} k^{-1 / 2}-2 a_{\mathrm{LR}}^{\prime} k^{-1 / 4} \gamma_{i}^{(j)}}{1+a_{\mathrm{LR}}^{\prime 2} k^{-1 / 2}-2 a_{\mathrm{LR}}^{\prime} k^{-1 / 4} \gamma_{i^{*}}^{(j)}} \geq\left(1+c \Delta^{2} k^{-1 / 2}\right)^{2}
$$

We now describe the intuition for the remaining argument. (10) is not hard to show when the unit vector $\widehat{\delta_{i}}$ is somewhat far from $\widehat{\delta_{i^{*}}}$, in which case it is reasonable to imagine a sizable cone of directions around $\delta_{i *}$ such that if $v_{j}$ lies in that cone, (10) holds. On the other hand, suppose $\widehat{\delta_{i}}$ is close to $\delta_{i^{*}}$. Then (10) can actually be false. But because their non-normalized counterparts $\delta_{i}$ and $\delta_{i^{*}}$ are assumed to be $\Delta$-separated, $\delta_{i}$ and $\delta_{i^{*}}$ must therefore be nearly collinear, in which case there must exist a gap between $\left\|\delta_{i}\right\|_{2}$ and $\left\|\delta_{i^{*}}\right\|_{2}$ that's even bigger than the one assumed in (7), and furthermore walking in $v_{j}$ cannot reduce this gap to below that of (7) in the next step.

We defer the formal details to the full version.

We now bound the probabilities of the events $A_{j}[i], B_{j}[i], C_{j}$. There are some minor complications from the fact that we don't have exact access to $\operatorname{span}\left(\left\{w_{i}-a_{t}\right\}\right)$ which we address now.

Define $\alpha_{\mathrm{svd}}^{(i)} \triangleq \frac{\left\|\mathrm{U} \delta_{i}\right\|_{2}}{\left\|\delta_{i}\right\|_{2}}$. By the second part of Lemma 6.2, $1-$ $\delta_{\text {samp }} \leq \alpha_{\mathrm{svd}}^{(i)} \leq 1$. First, for any $i \in[k], \gamma_{i}^{(j)}=\alpha_{\mathrm{svd}}^{(i)}\left\langle\frac{g}{\|g\|_{2}}, \frac{\mathbf{U} \delta_{i}}{\left\|\mathbf{U} \delta_{i}\right\|_{2}}\right\rangle$, where $g \sim \mathcal{N}\left(0, \mathrm{I}_{k}\right)$ and the last step follows by the second part of Lemma 6.2. The random variable $\left\langle\frac{g}{\|g\|_{2}}, \frac{\mathbf{U} \delta_{i}}{\left\|\mathbf{U} \delta_{i}\right\|_{2}}\right\rangle$ is merely the correlation of a random unit vector with a fixed unit vector; call this random variable $X$ (clearly it does not depend on the fixed vector).

CLAIM 6.12. For any $j \in[M], \operatorname{Pr}\left[A_{j}\left[i^{*}\right]\right] \geq e^{-a_{\text {trials }} \sqrt{k} / \Delta^{2}}$ for some absolute constant $a_{\text {trials }}>0$.

Claim 6.13. For any $i \in[k]$ and $j \in[M], \operatorname{Pr}\left[B_{j}[i]\right] \geq 1-$ $e^{-\overline{a_{\text {trials }}} \sqrt{k} / \Delta^{2}}$ for some absolute constant $\overline{a_{\text {trials }}}>a_{\text {trials }}$.

We next lower bound the probability of event $C_{j}$ relative to that of $A_{j}\left[i^{*}\right]$. Equivalently, provided $A_{j}\left[i^{*}\right]$ happens, we lower bound the conditional probability that the gap of (7) is preserved. In this proof, we would like to use the fact that $\delta_{i}^{\perp}$ is orthogonal to $\widehat{\delta}_{i^{*}}$ for all $i \neq i^{*}$ to argue that $\left\langle g \mathbf{U}, \delta_{i}^{\perp}\right\rangle$ and $\left\langle g \mathrm{U}, \delta_{i^{*}}\right\rangle$ are independent. Again, this is only true if $\mathbf{U}$ is exactly the projector to the span of $\left\{w_{i}-a_{t}\right\}$, and we need to argue that it suffices to take $\mathbf{U}$ an approximation to that projector.

Claim 6.14. For any $j \in[M], \operatorname{Pr}\left[C_{j}\right] \geq \frac{1}{\operatorname{poly}(k)} \operatorname{Pr}\left[A_{j}\left[i^{*}\right]\right]$.

The proof is somewhat technical and we defer it to the full version. Next, we would like to show that for any $j \in[M]$, if we condition on the events $B_{j}[i]$ holding for all $i \in[k]$, then the conditional probability of $A_{j}[i]$ is not much more than that of $A_{j}\left[i^{*}\right]$. Note that by rotational invariance, these conditional probabilities would be identical if $\mathbf{U}$ were exactly the projector to the span of $\left\{w_{i}-a_{t}\right\}$, and here it is straightforward to see that it suffices to take $\mathrm{U}$ a sufficiently good approximation to that projector.

Claim 6.15. For any $j \in[M]$ and $i \neq i^{*}$, if $\delta_{\text {samp }}=1 / \operatorname{poly}(k)$ is sufficiently small, $\operatorname{Pr}\left[A_{j}[i] \wedge B_{j}\right] \leq k \cdot \operatorname{Pr}\left[A_{j}\left[i^{*}\right] \wedge B_{j}\right]$. 
We can now combine these bounds to show that if COMPAREMINVAR succeeds and outputs true on some $v$, the conditional probability that the gap of (7) has been preserved is at least $1 / \operatorname{poly}(k)$.

CLAIM 6.16. Let $B_{v}$ be the event that $\operatorname{Pr}\left[\left\langle\widehat{\delta}_{i}, v\right\rangle\right] \leq v_{B} \Delta k^{-1 / 4}$ for all $i \in[k]$. Let detect - progress $_{v}$ be the event that $B_{v}$ occurs and additionally that COMPAREMINVAR succeeds and outputs true on direction $v$. Let gap - preserved ${ }_{v}$ be the event that $B_{v}$ occurs and additionally $\left\|\delta_{i}-\eta v\right\|_{2} \geq\left(1+c \Delta^{2} k^{-1 / 2}\right) \cdot\left\|\delta_{i^{*}}-\eta v\right\|_{2}$ for all $i \neq i^{*}$. Then $\operatorname{Pr}_{v}\left[\right.$ gap $-\operatorname{preserved}_{v} \mid$ detect $\left.-\operatorname{progress}_{v}\right] \geq 1 / \operatorname{poly}(k)$.

We are now ready to finish the proof of Lemma 6.4. First, condition on the event that all $M$ runs of COMPAREMinVAR are successful, which happens with probability at least $1-\delta / 3$. The probability that $B_{j}[i]$ holds for all $i \in[k], j \in[M]$ is at least $1-k M e^{-\overline{a_{\text {trials }}} \sqrt{k} / \Delta^{2}}$, by Claim 6.13. Condition on this happening. The probability that detect - $\operatorname{progress}_{v_{j}}$ occurs for some $j \in[M]$ is at least the probability that $A_{j}\left[i^{*}\right]$ occurs for some $j \in[M]$, and this is at least $1-\left(1-e^{-a_{\text {trials }} \sqrt{k} / \Delta^{2}}\right)^{M} \geq 1-e^{-M e^{-a_{\text {trials }} \sqrt{k} / \Delta^{2}}}$ by Claim 6.12. So by taking $M=e^{-a_{\text {trials }} \sqrt{k} / \Delta^{2}} \ln (3 / \delta)$, by a union bound we conclude that with probability at least $1-\delta$, every run of COMPAREMINVAR succeeds, $B_{j}[i]$ holds for every $i \in[k], j \in[M]$, and furthermore there is some $j$ for which detect - progress $_{v_{j}}$ occurs.

If detect - $\operatorname{progress}_{v_{j}}$ occurs for some $j$, then for that particular $j$, gap - $\operatorname{preserved}_{v_{j}}$ holds with probability at least $1 / \operatorname{poly}(k)$.

\subsection{Initializing With a Gap}

A key assumption in Lemma 6.4 is that there is a gap between $\left\|w_{i^{*}}-a_{t}\right\|_{2}$ and all other $\left\|w_{i}-a_{t}\right\|_{2}$. We next show that this assumption can be made to hold when $t=0$. The high-level structure of the proof will be very similar to that of Lemma 6.11.

LEMma 6.17. There is a constant $\tau_{\text {gap }}^{\prime}>0$ such that for all $c^{\prime}<$ $\tau_{\text {gap }}^{\prime}$, the following holds for any sufficiently small $v_{*}=\operatorname{poly}(\Delta, 1 / k)$.

Fix any $i^{*} \in[k]$ and suppose that $\left\|w_{i^{*}}\right\|_{2} \geq \underline{\sigma}$ for some $\underline{\sigma}>0$. Let

$$
\mathcal{S} \triangleq\left\{\underline{\sigma} \cdot k^{1 / 4}, \underline{\sigma} \cdot\left(1+v_{*}\right) \cdot k^{1 / 4}, \underline{\sigma} \cdot\left(1+v_{*}\right)^{2} \cdot k^{1 / 4}, \ldots, k^{1 / 4}\right\} .
$$

Then any $i \neq i^{*}$ and $v \in \mathbb{R}^{d}$, let $\mathcal{E}_{v}[i]$ denote the event that

$$
\left\|w_{i}-v\right\|_{2}^{2} \geq\left(1+c^{\prime} \cdot \frac{\Delta^{2}}{\sqrt{k}}\right) \cdot\left\|w_{i^{*}}-v\right\|_{2}^{2}
$$

and define $\mathcal{E}_{v}$ to be the event that $\mathcal{E}_{v}[i]$ occurs simultaneously for all $i \neq i^{*}$. There exists $\alpha \in \mathcal{S}$ for which $\operatorname{Pr}_{\|v\|_{2}=\alpha}\left[\mathcal{E}_{v}\right] \geq$ $\exp \left(-O\left(\sqrt{k} / \Delta^{2}\right)\right)$, where the probability is over $v$ a Haar-random vector in $\mathbb{R}^{d}$ with norm $\alpha$.

Proof. By design, there exists an $\alpha \in \mathcal{S}$ for which $\alpha=(1+v)$. $\left\|w_{i^{*}}\right\|_{2} \cdot k^{1 / 4}$ for $v \in\left[-v_{*}, v_{*}\right]$. Let $v$ be random with norm $\alpha$.

Define $\widehat{w}_{i}=w_{i} /\left\|w_{i}\right\|_{2}$. For every $i \neq i^{*}$, define $w_{i}^{\perp} \triangleq \widehat{w}_{i}-$ $\left\langle\widehat{w}_{i^{*}}, \widehat{w}_{i}\right\rangle \widehat{w}_{i^{*}}$. Repurposing notation from the proof of Lemma 6.4, let $\gamma_{i}=\left\langle\widehat{w}_{i}, v /\|v\|_{2}\right\rangle$. Also, let $\rho_{i} \triangleq\left\|w_{i}\right\|_{2} /\left\|w_{i^{*}}\right\|_{2}$. Under this,

$$
\begin{aligned}
\frac{\left\|w_{i}-v\right\|_{2}^{2}}{\left\|w_{i^{*}}-v\right\|_{2}^{2}} & =\frac{\left\|w_{i}\right\|_{2}^{2}+\alpha^{2}-2 \alpha \gamma_{i}\left\|w_{i}\right\|_{2}}{\left\|w_{i^{*}}\right\|_{2}^{2}+\alpha^{2}-2 \alpha \gamma_{i^{*}}\left\|w_{i^{*}}\right\|_{2}} \\
& =\frac{\rho_{i}^{2}+(1+v)^{2} \sqrt{k}-2(1+v) \rho_{i} k^{1 / 4} \gamma_{i}}{1+(1+v)^{2} \sqrt{k}-2(1+v) k^{1 / 4} \gamma_{i^{*}}} .
\end{aligned}
$$

Using similar terminology as in the proof of Lemma 6.4, define the following two types of events over the random vector $v$ :

(1) Let $B[i]$ be the event that $\gamma_{i} \leq k^{-1 / 4} \cdot\left(1+c \Delta^{2}\right)$ for some absolute constant $c>0$.

(2) Let $C$ be the event $\gamma_{i^{*}} \geq k^{-1 / 4}$ and $\left\langle v, w_{i}^{\perp}\right\rangle \leq v_{\perp} k^{-1 / 2}\left\|w_{i}^{\perp}\right\|_{2}$ for all $i \neq i^{*}$, for some absolute constant $v_{\perp}$.

The main step will be to show that these events imply $\mathcal{E}_{v}$.

Claim 6.18. Let $i \neq i^{*}$. If $B[i]$ and $C$ occur, then $\mathcal{E}_{v}[i]$ occurs.

The proof of this is very technical, so we defer it to the full version. To complete the proof, we must show that the probability $B[i]$ and $C$ occur simultaneously for all $i \in[k]$ is at least $\exp \left(-O\left(\sqrt{k} / \Delta^{2}\right)\right)$. The proofs for these facts are essentially identical to those of Claims 6.12, 6.13, and 6.14 in the proof of Lemma 6.4..

Claim 6.19. For any $i \in[k], \operatorname{Pr}[B[i]] \geq 1-e^{-\bar{a} \sqrt{k} / \Delta^{2}}$ for some absolute constant $\bar{a}>0$.

CLAIM 6.20. For any $i \in[k], \operatorname{Pr}[C] \geq \frac{1}{\operatorname{poly}(k)} e^{-\underline{a} \sqrt{k} / \Delta^{2}}$ for some absolute $\underline{a}>0$ s.t. $\bar{a}-\underline{a}$ is nonnegative and strictly increasing in $\Delta$.

Lemma 6.17 now follows by a union bound: the probability that all $B[i]$ occur is at least $1-k \cdot e^{-\bar{a} \sqrt{k} / \Delta^{2}}$, and the probability that $C$ occurs is $\frac{1}{\operatorname{poly}(k)} e^{-\underline{a} \sqrt{k} / \Delta^{2}}$, so the probability all of these events occur is at least $\frac{1}{\text { poly }(k)} e^{-\underline{a} \sqrt{k} / \Delta^{2}}-k \cdot e^{-\bar{a} \sqrt{k} / \Delta^{2}}=\exp \left(-O\left(\sqrt{k^{2}} / \Delta\right)\right)$, and then we are done by Claim 6.18.

Lastly, we remark that Lemma 6.17 only applies to $w_{i^{*}}$ for which $\left\|w_{i^{*}}\right\|_{2} \geq \underline{\sigma}$. We could take $\underline{\sigma}=\epsilon / 4$ at no cost to the asymptotics of our runtime. Now for regressors $w_{i}$ whose norm is less than $\epsilon / 4$, we can simply output an arbitrary vector $a$ of norm $\epsilon / 4$ as an $\epsilon / 2$-close estimate, by triangle inequality. We can also easily check whether there is indeed such a short regressor $w_{i}$, e.g. by estimating the minimum variance of the univariate mixture $\mathcal{F}$ given by sampling $(x, y) \sim \mathcal{D}$ and computing $y-\langle a, x\rangle$ (see CнескOutcome).

\subsection{Algorithm Specification}

Due to space constraints, we defer a full specification of our algorithm LEARNWithNoise to the full version. At a high level, we will run FourierMomentDescent $\exp \left(O\left(\sqrt{k} / \Delta^{2}\right)\right)$ times under random initializations as specified by Lemma 6.17. Based on what we have shown, with high probability we will get at least one estimate for every component, and it is not hard to certify whether the component learned in any given iteration is distinct from the components already learned by computing the appropriate residual and invoking the technology of Section 5.1.

\section{LEARNING MIXTURES OF HYPERPLANES}

In this section we show that our techniques extend to give a subexponential time algorithm for learning mixtures of hyperplanes. Formally, we show the following:

Theorem 7.1. Given $\delta, \epsilon>0$ and a mixture of hyperplanes $\mathcal{D}$ with directions $\left\{v_{1}, \ldots, v_{k}\right\}$, separation $\Delta$, with probability at least $1-\delta$, $\operatorname{LEARNPLANES}(\mathcal{D}, \delta, \epsilon)$ returns a list of unit vectors $\mathcal{L} \triangleq\left\{\widetilde{v}_{1}, \ldots, \widetilde{v}_{k}\right\}$ for which there is a permutation $\pi:[k] \rightarrow[k]$ and signs $\epsilon_{1}, \ldots, \epsilon_{k} \in$ $\{ \pm 1\}$ for which $\left\|\widetilde{v}_{i}-\epsilon_{i} v_{\pi(i)}\right\|_{2} \leq \epsilon$ for all $i \in[k]$. Furthermore, 
it requires $N=\widetilde{O}\left(d \ln (1 / \epsilon) \ln (1 / \delta) p_{\min }^{-4} \Delta^{-2} \cdot \operatorname{poly}\left(k, \ln \left(1 / p_{\min }\right)\right.\right.$, $\left.\ln (1 / \Delta))^{O\left(k^{3 / 5} \ln \left(1 / p_{\min }\right)\right)}\right)$ samples and time $N d \cdot \operatorname{poly} \log (k, d, 1 / \Delta$, $\left.1 / p_{\min }, 1 / \epsilon\right)$ time.

In Section 7.1 we show the key fact that a random step will contract $\min _{i}\left\|\Pi_{i} a_{t}\right\|_{2}$ by a factor of $1-\Theta\left(k^{-3 / 5}\right)$ with probability at least $\exp \left(-k^{3 / 5}\right)$, provided we use a suitable initialization. In Section 7.2 we describe our algorithm HyperplaneMomentDescent for learning a single component in a mixture of hyperplanes and sketch how it can be used to learn the full mixture.

\subsection{Moment Descent for Hyperplanes}

In this section we give the key technical ingredients for showing that a suitable modification of FourierMomentDescent (Algorithm 4) can also be used to learn mixtures of hyperplanes.

Similar to the case of MLRs, here the first step is to estimate the span of the directions $\left\{v_{i}\right\}$. Define the matrix $\mathbf{M} \triangleq \mathbf{I}-\mathbb{E}_{x \sim \mathcal{D}}\left[x x^{\top}\right]=$ $\sum_{i=1}^{k} p_{i} v_{i} v_{i}^{\top}$ and let $\widehat{\mathbf{M}}^{(N)} \triangleq \mathbf{I}-\frac{1}{N} \sum_{i=1}^{N} x_{i} x_{i}^{\top}$ for $x_{1}, \ldots, x_{N}$ i.i.d samples from $\mathcal{D}$. We will often omit the superscript $(N)$.

For any $\delta_{\text {samp }}, \delta>0$, if $N=\widetilde{\Omega}\left(d \cdot \ln (1 / \delta) \cdot p_{\text {min }}^{-4} \cdot \delta_{\text {samp }}^{-2}\right)$, then by standard concentration, $\left\|\mathbf{M}-\widehat{\mathbf{M}}^{(N)}\right\|_{2} \leq p_{\min } \cdot \delta_{\text {samp }} / 2$ with probability at least $1-\delta$

Henceforth, let $\mathbf{W} \in \mathbb{R}^{k \times d}$ be the matrix whose rows are the top $k$ singular vectors of $\mathbf{M}$, let $\mathbf{U} \in \mathbb{R}^{k \times d}$ be the matrix whose rows are the first $k$ singular vectors of $\widehat{\mathbf{M}}^{(N)}$ for the above choice of $N$.

Lemma 7.2. Let $a_{t} \in \mathbb{S}^{d-1}$ lie in the row span of $\mathrm{U}$, let $v_{j}=$ $x_{j} /\left\|x_{j}\right\|_{2}$, and let $\Pi_{j}$ be the projector to the orthogonal complement of $v_{j}$. Suppose $\delta_{\text {samp }}<1 / k$ and $\left\|x_{j}\right\| \leq 1$. Then $\left\|\mathrm{U} \Pi_{j} a_{t}\right\|_{2} \geq\left\|\Pi_{j} a_{t}\right\|_{2}-$

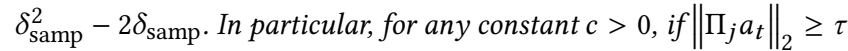
for some $\tau=\operatorname{poly}(k)$, then for sufficiently small $\delta_{\text {samp }}=1 / \operatorname{poly}(k)$ we can ensure that $\left\|\mathrm{U}_{j} a_{t}\right\|_{2} \geq\left(1-k^{-c}\right)\left\|\Pi_{j} a_{t}\right\|$.

The proof of this is straightforward, see the full version. We can now prove the main result of this section, the mixture of hyperplanes analogue of Lemma 5.12.

Lemma 7.3. Let $0<c<1 / 2$. There are absolute constants $\alpha>$ $0, \beta>1, v>0$ such that for any $\delta>\exp \left(-v \cdot k^{1-2 c}\right)$, the following holds for $k$ sufficiently large. Let $\sigma_{t} \triangleq \min _{i \in[k]}\left\|\Pi_{i} a_{t}\right\|_{2}$, and suppose $\delta_{\text {samp }} \leq \sigma_{t}^{2} / 9$. Denote the minimizing index $i$ by $i^{*}$. For $M \triangleq$ $e^{-v \cdot k^{1-2 c}} \ln (2 / \delta)$ and $g_{1}, \ldots, g_{M} \sim \mathcal{N}\left(0, \mathbf{I}_{k}\right)$, let $z_{j}=\frac{g_{j} \mathrm{U}}{g_{j}\|\mathbf{U}\|_{2}} \in \mathbb{S}^{d-1}$ for $j \in[M]$. Let $\sigma^{*}$ satisfy $0.9 \sigma_{t} \leq \sigma^{*} \leq 1.1 \sigma_{t}$. Let $\eta=k^{-c} \sigma^{*}$.

If $\left|\left\langle a_{t}, v_{i^{*}}\right\rangle\right| \geq k^{-c}$, then 1) there exists at least one $j \in[M]$, call it $j^{*}$, for which $\left\|\Pi_{i^{*}}\left(a_{t}-\eta \cdot z_{j}\right)\right\|_{2}^{2} \leq\left(1-\frac{\alpha}{k^{3 c}}\right) \sigma_{t}^{2}$, and 2) for all $j \in[M]$ and $i \in[k], \frac{\left\|\Pi_{i}\left(a_{t}-\eta z_{j}\right)\right\|_{2}^{2}}{\sigma_{t}^{2}} \geq\left(\frac{\left\|\Pi_{i^{*}}\left(a_{t}-\eta z_{j^{*}}\right)\right\|_{2}^{2}}{\sigma_{t}^{2}}\right)^{\beta}$, all with probability at least $1-\delta$.

Proof. Suppose $\left\langle a_{t}, v_{i^{*}}\right\rangle \geq 0$. For $j \in[M]$, let $\omega_{j} \triangleq-\eta^{2}+$ $2 \eta\left\langle z_{j}, \Pi_{i} a_{t}\right\rangle$ and $a_{t+1}^{(j)}=a_{j}-\eta \cdot z_{j}$. One can show that

$$
\frac{\left\|\Pi_{i} a_{t+1}^{(j)}\right\|_{2}^{2}}{\left\|\Pi_{i} a_{t}\right\|_{2}^{2}}=\frac{1-\left\|\Pi_{i} a_{t}\right\|_{2}^{-2}\left(\omega_{j}+\eta^{2}\left\langle v_{i}, z_{j}\right\rangle^{2}\right)}{1-\omega_{j}-2 \eta\left\langle a_{t}, v_{i}\right\rangle\left\langle z_{j}, v_{i}\right\rangle} .
$$

Define the events

$$
\begin{gathered}
A_{j} \triangleq\left\{\left\langle z_{j}, \Pi_{i^{*}} a_{t}\right\rangle \geq k^{-c}\left\|\Pi_{i^{*}} a_{t}\right\|_{2} \quad \text { and }\left\langle z_{j}, v_{i^{*}}\right\rangle \leq-k^{-c}\right\} . \\
B_{j}[i] \triangleq\left\{\left|\left\langle z_{j}, \Pi_{i} a_{t}\right\rangle\right| \leq \xi k^{-c}\left\|\Pi_{i} a_{t}\right\|_{2} \text { and }\left|\left\langle z_{j}, v_{i}\right\rangle\right| \leq \xi k^{-c}\right\}
\end{gathered}
$$

for some $\xi>1$ to be specified later. We show in Claim 7.4 below that for any given $j$, there is some absolute constant $v>0$ such that $\operatorname{Pr}\left[A_{j}\right] \geq \exp \left(-v \cdot k^{1-2 c}\right)$, and some absolute constant $\xi>1$ such that $\operatorname{Pr}\left[B_{j}[i]\right] \geq 1-\exp \left(-3 v \cdot k^{1-2 c}\right)$.

We will now argue that $A_{j}$ corresponds to making good progress, while $B_{j}[i]$ corresponds to not making too much progress.

Suppose $A_{j}$ held for some $j=j^{*}$ and $B_{j}[i]$ held for all $i \in[k], j \in$ [M]. If we took $\eta \triangleq k^{-c} \sigma^{*}$, we would conclude from the definition of $A_{j^{*}}$ and the assumption $0.9 \sigma_{t} \leq \sigma^{*} \leq 1.1 \sigma_{t}$ that

$$
\omega_{j^{*}} \geq\left\|\Pi_{i^{*}} a_{t}\right\|_{2}^{2} k^{-2 c} \cdot\left(-0.9^{2}+2 \cdot 0.9\right)=0.99\left\|\Pi_{i^{*}} a_{t}\right\|_{2}^{2} k^{-2 c} .
$$

Likewise from the definition of $B_{j}[i]$ we would conclude that

$$
\omega_{j} \leq\left\|\Pi_{i} a_{t}\right\|_{2}^{2} k^{-2 c} \cdot(-1.21+2.2 \xi) .
$$

for all $j \in[M]$ and $i \in[k]$. One can show (see the full version), for the functions $\bar{g}\left(\left\langle a_{t}, v_{i}\right\rangle\right) \triangleq \frac{1}{0.99}\left(1.21 \xi^{2} k^{-4 c}+(2.2 \xi-\right.$ $\left.1.21) k^{-2 c}\left\langle a_{t}, v_{i}\right\rangle^{2}+1.8 \xi k^{-2 c}\left\|\Pi_{i} a_{t}\right\|_{2}\left\langle a_{t}, v_{i}\right\rangle\right)$ and $g\left(\left\langle a_{t}, v_{i^{*}}\right\rangle\right) \triangleq$ $\frac{1}{1.01}\left(0.81 k^{-4 c}+0.99 k^{-2 c}\left\langle a_{t}, v_{i^{*}}\right\rangle^{2}+2.2 k^{-2 c}\left\|\Pi_{i^{*}} a_{t}\right\|_{2}\left\langle a_{t}, v_{i^{*}}\right\rangle\right)$, that $1-\left\|\Pi_{i} a_{t+1}^{(j)}\right\|_{2}^{2} /\left\|\Pi_{i} a_{t}\right\|_{2}^{2} \leq \bar{g}\left(\left\langle a_{t}, v_{i}\right\rangle\right)$ for every $j \in[M], i \in[k]$, and for $i=i^{*}, j=j^{*}$, we have $1-\left\|\Pi_{i^{*}} a_{t+1}^{\left(j^{*}\right)}\right\|_{2}^{2} /\left\|\Pi_{i^{*}} a_{t}\right\|_{2}^{2} \geq g\left(\left\langle a_{t}, v_{i^{*}}\right\rangle\right)$.

To control these quantities, note that the function $g(\bar{x})$ is increasing over the interval $\left[0, \tau^{*}\right]$ and decreasing over the interval $\left[\tau^{*}, 1\right]$ for some constant $\tau^{*} \in[0.91,0.92]$. When $\left\langle v_{i^{*}}, a_{t}\right\rangle=1$, we get that $g=\Omega\left(k^{-2 c}\right)$, because the $0.99 k^{-2 c}\left\langle a_{t}, v_{i^{*}}\right\rangle^{2}$ term in the definition of $\underline{g}$ dominates. And when $\left\langle v_{i^{*}}, a_{t}\right\rangle=k^{-c}$, we get that $\underline{g}=\Omega\left(k^{-3 c}\right)$, because the $2.2 k^{-2 c}\left\|\Pi_{i^{*}} a_{t}\right\|_{2}\left\langle a_{t}, v_{i^{*}}\right\rangle$ term in the definition of $\underline{g}$ dominates. So the first part of the lemma follows.

On the other hand, there is some absolute constant $\beta^{\prime}>1$ such that $g(x) \leq \bar{g}(x) \leq \beta^{\prime} g(x)$ for all $x \in[0,1]$. There is some constant $\beta^{\prime \prime}>1$ for which $g(x) / g(y)<\beta^{\prime \prime}$ for all $0 \leq x \leq y \leq 1$. The reason is that $g(x)$ is increasing over the interval $\left[0, \tau^{*}\right]$ and decreasing over the interval $\left[\tau^{*}, 1\right]$, and $g(x)=1-\Omega\left(k^{-2 c}\right)$ for $x \in\left[\tau^{*}, 1\right]$.

It follows that for any $j \in \overline{[} M], i \in[k]$

$$
1-\frac{\left\|\Pi_{i} a_{t+1}^{(j)}\right\|_{2}^{2}}{\left\|\Pi_{i} a_{t}\right\|_{2}^{2}} \leq \beta^{\prime} \beta^{\prime \prime} \cdot\left(1-\frac{\left\|\Pi_{i^{*}} a_{t+1}^{\left(j^{*}\right)}\right\|_{2}^{2}}{\left\|\Pi_{i^{*}} a_{t}\right\|_{2}^{2}}\right),
$$

so by taking $\beta$ in the statement of the lemma to be $\beta^{\prime} \cdot \beta^{\prime \prime}$ and invoking the elementary inequality $1-a \cdot x \leq(1-x)^{a}$ for $a>1$, we get the second part of the lemma.

We conclude that if $A_{j}$ held for some $j \in[M]$ and $B_{j}[i]$ held for all $j \in[M], i \in[k]$, then both parts of Lemma 7.3 would hold. From Claims 7.4 below, whose proof we defer to the full version, it is not hard to upper bound the probability this does not happen.

CLAIM 7.4. There are absolute constants $v>0, \xi>1$ such that for any $j \in[M], i \in[k], \operatorname{Pr}\left[A_{j}\right] \geq \exp \left(-v \cdot k^{1-2 c}\right)$ and $\operatorname{Pr}\left[B_{j}[i]\right] \geq$ $1-\exp \left(-3 v \cdot k^{1-2 c}\right)$. 


\subsection{Algorithm Specification}

Similar to LEARNWITHNoISE sketched in Section 6, Lemma 7.3 requires the initialization $a_{0}$ to satisfy $\left|\left\langle a_{0}, v_{i^{*}}\right\rangle\right|$, but apart from this, the algorithm essentially proceeds in the same way as FourierMomentDescent to get a warm start for a single component. We can then use the boosting procedure of [30] to boost the warm start to arbitrary accuracy and peel off this component and recurse. Instantiating the boosting of [30] in the setting of mixtures of hyperplanes is slightly nontrivial, and we defer all the details, including the runtime analysis, to the full version.

\section{ACKNOWLEDGMENTS}

S.C. and J.L. would like to thank Sam Hopkins and Tselil Schramm for answering questions regarding low-degree hypothesis testing, and Ankur Moitra for helpful discussions early on in this project.

\section{REFERENCES}

[1] Jayadev Acharya, Ilias Diakonikolas, Jerry Li, and Ludwig Schmidt. 2017. Sampleoptimal density estimation in nearly-linear time. In Proceedings of the TwentyEighth Annual ACM-SIAM Symposium on Discrete Algorithms. SIAM, 1278-1289.

[2] Laurent Bako. 2011. Identification of switched linear systems via sparse optimization. Automatica 47, 4 (2011), 668-677.

[3] Sivaraman Balakrishnan, Martin J Wainwright, and Bin Yu. 2017. Statistical guarantees for the EM algorithm: From population to sample-based analysis. The Annals of Statistics 45, 1 (2017), 77-120.

[4] Radu Balan, Pete Casazza, and Dan Edidin. 2006. On signal reconstruction without phase. Applied and Computational Harmonic Analysis 20, 3 (2006), 345-356.

[5] Emmanuel J Candes, Thomas Strohmer, and Vladislav Voroninski. 2013. Phaselift Exact and stable signal recovery from magnitude measurements via convex programming. Communications on Pure and Applied Mathematics 66, 8 (2013), 1241-1274.

[6] Arun Tejasvi Chaganty and Percy Liang. 2013. Spectral experts for estimating mixtures of linear regressions. In International Conference on Machine Learning. 1040-1048.

[7] Siu-On Chan, Ilias Diakonikolas, Rocco A Servedio, and Xiaorui Sun. 2014. Efficient density estimation via piecewise polynomial approximation. In Proceedings of the forty-sixth annual ACM symposium on Theory of computing. ACM, 604-613.

[8] Moses Charikar, Jacob Steinhardt, and Gregory Valiant. 2017. Learning from untrusted data. In Proceedings of the 49th Annual ACM SIGACT Symposium on Theory of Computing. ACM, 47-60.

[9] Xue Chen, Daniel M Kane, Eric Price, and Zhao Song. 2016. Fourier-sparse interpolation without a frequency gap. In Foundations of Computer Science (FOCS), 2016 IEEE 57th Annual Symposium on. IEEE, 741-750.

[10] Yudong Chen, Xinyang Yi, and Constantine Caramanis. 2013. A convex formulation for mixed regression with two components: Minimax optimal rates. arXiv preprint arXiv:1312.7006 (2013).

[11] Richard D De Veaux. 1989. Mixtures of linear regressions. Computational Statistics \& Data Analysis 8, 3 (1989), 227-245.

[12] Ilias Diakonikolas. 2016. Learning Structured Distributions. Handbook of Big Data 267 (2016).

[13] Ilias Diakonikolas, Daniel M Kane, and Alistair Stewart. 2016. The fourier transform of poisson multinomial distributions and its algorithmic applications. In Proceedings of the forty-eighth annual ACM symposium on Theory of Computing. ACM, 1060-1073.

[14] Ilias Diakonikolas, Daniel M Kane, and Alistair Stewart. 2016. Optimal learning via the fourier transform for sums of independent integer random variables. In Conference on Learning Theory. 831-849.

[15] Ilias Diakonikolas, Daniel M Kane, and Alistair Stewart. 2016. Properly learning poisson binomial distributions in almost polynomial time. In Conference on Learning Theory. 850-878.

[16] Ehsan Elhamifar and Rene Vidal. 2013. Sparse subspace clustering: Algorithm, theory, and applications. IEEE transactions on pattern analysis and machine intelligence 35, 11 (2013), 2765-2781.

[17] Susana Faria and Gilda Soromenho. 2010. Fitting mixtures of linear regressions. fournal of Statistical Computation and Simulation 80, 2 (2010), 201-225.

[18] Martin A Fischler and Robert C Bolles. 1981. Random sample consensus: a paradigm for model fitting with applications to image analysis and automated cartography. Commun. ACM 24, 6 (1981), 381-395.

[19] Scott Gaffney and Padhraic Smyth. 1999. Trajectory clustering with mixtures of regression models. In Proceedings of the fifth ACM SIGKDD international conference on Knowledge discovery and data mining. ACM, 63-72.
[20] Haitham Hassanieh, Piotr Indyk, Dina Katabi, and Eric Price. 2012. Nearly optimal sparse Fourier transform. In Proceedings of the forty-fourth annual ACM symposium on Theory of computing. ACM, 563-578.

[21] Nina Holden, Yuval Peres, and Alex Zhai. 2018. Gravitational allocation on the sphere. Proceedings of the National Academy of Sciences 115, 39 (2018), 9666-9671.

[22] Piotr Indyk and Michael Kapralov. 2014. Sample-optimal Fourier sampling in any constant dimension. In Foundations of Computer Science (FOCS), 2014 IEEE 55th Annual Symposium on. IEEE, 514-523.

[23] Michael I Jordan and Robert A Jacobs. 1994. Hierarchical mixtures of experts and the EM algorithm. Neural computation 6, 2 (1994), 181-214.

[24] Michael Kapralov. 2016. Sparse Fourier Transform in Any Constant Dimension with Nearly-Optimal Sample Complexity in Sublinear Time. In Symposium on Theory of Computing Conference, STOC'16, Cambridge, MA, USA, June 19-21, 2016.

[25] Michael Kapralov. 2017. Sample Efficient Estimation and Recovery in Sparse FFT via Isolation on Average. In Foundations of Computer Science, 2017. FOCS'17. IEEE 58th Annual IEEE Symposium on. https://arxiv.org/pdf/1708.04544.

[26] Sushrut Karmalkar, Pravesh Kothari, and Adam Klivans. 2019. List-Decodable Linear Regression. In NeurIPS. arXiv preprint arXiv:1905.05679.

[27] Jason M Klusowski, Dana Yang, and WD Brinda. 2017. Estimating the coefficients of a mixture of two linear regressions by expectation maximization. arXiv preprint arXiv:1704.08231 (2017).

[28] Jeongyeol Kwon and Constantine Caramanis. 2019. EM Converges for a Mixture of Many Linear Regressions. arXiv preprint arXiv:1905.12106 (2019).

[29] Jeongyeol Kwon, Wei Qian, Constantine Caramanis, Yudong Chen, and Damek Davis. 2018. Global convergence of EM algorithm for mixtures of two component linear regression. arXiv preprint arXiv:1810.05752 (2018).

[30] Yuanzhi Li and Yingyu Liang. 2018. Learning Mixtures of Linear Regressions with Nearly Optimal Complexity. In Conference On Learning Theory. 1125-1144.

[31] Guangcan Liu, Zhouchen Lin, Shuicheng Yan, Ju Sun, Yong Yu, and Yi Ma. 2012. Robust recovery of subspace structures by low-rank representation. IEEE transactions on pattern analysis and machine intelligence 35, 1 (2012), 171-184.

[32] Can-Yi Lu, Hai Min, Zhong-Qiu Zhao, Lin Zhu, De-Shuang Huang, and Shuicheng Yan. 2012. Robust and efficient subspace segmentation via least squares regression. In European conference on computer vision. Springer, 347-360.

[33] Ankur Moitra. 2015. The threshold for super-resolution via extremal functions. In STOC.

[34] Ankur Moitra and Gregory Valiant. 2010. Settling the polynomial learnability of mixtures of gaussians. In Foundations of Computer Science (FOCS), 2010 51st Annual IEEE Symposium on. IEEE, 93-102.

[35] Vasileios Nakos, Zhao Song, and Zhengyu Wang. 2019. (Nearly) Sample-Optimal Sparse Fourier Transform in Any Dimension; RIPless and Filterless. In FOCS.

[36] Praneeth Netrapalli, Prateek Jain, and Sujay Sanghavi. 2013. Phase retrieval using alternating minimization. In Advances in Neural Information Processing Systems. 2796-2804.

[37] Lance Parsons, Ehtesham Haque, and Huan Liu. 2004. Subspace clustering for high dimensional data: a review. Acm Sigkdd Explorations Newsletter 6, 1 (2004), 90-105.

[38] Eric Price and Zhao Song. 2015. A Robust Sparse Fourier Transform in the Continuous Setting. In Foundations of Computer Science (FOCS), 2015 IEEE 56th Annual Symposium on. IEEE, 583-600.

[39] Prasad Raghavendra and Morris Yau. 2019. List Decodable Learning via Sum of Squares. arXiv preprint arXiv:1905.04660 (2019).

[40] Hanie Sedghi, Majid Janzamin, and Anima Anandkumar. 2016. Provable tensor methods for learning mixtures of generalized linear models. In Artificial Intelligence and Statistics. 1223-1231.

[41] Manolis C Tsakiris and René Vidal. 2015. Dual principal component pursuit. In Proceedings of the IEEE International Conference on Computer Vision Workshops. $10-18$.

[42] Manolis C Tsakiris and René Vidal. 2017. Hyperplane clustering via dual principal component pursuit. In Proceedings of the 34th International Conference on Machine Learning-Volume 70. JMLR. org, 3472-3481.

[43] René Vidal. 2011. Subspace clustering. IEEE Signal Processing Magazine 28, 2 (2011), 52-68.

[44] Rene Vidal and Richard Hartley. 2007. Three-view multibody structure from motion. IEEE transactions on pattern analysis and machine intelligence 30,2 (2007), 214-227.

[45] Rene Vidal, Yi Ma, and Shankar Sastry. 2005. Generalized principal component analysis (GPCA). IEEE transactions on pattern analysis and machine intelligence 27, 12 (2005), 1945-1959.

[46] Xinyang Yi, Constantine Caramanis, and Sujay Sanghavi. 2014. Alternating minimization for mixed linear regression. In International Conference on Machine Learning. 613-621.

[47] Xinyang Yi, Constantine Caramanis, and Sujay Sanghavi. 2016. Solving a mixture of many random linear equations by tensor decomposition and alternating minimization. arXiv preprint arXiv:1608.05749 (2016).

[48] Kai Zhong, Prateek Jain, and Inderjit S Dhillon. 2016. Mixed linear regression with multiple components. In Advances in neural information processing systems. 2190-2198. 